ARTICLE

https://doi.org/10.1038/s41467-019-10379-7

\title{
FOXF2 reprograms breast cancer cells into bone metastasis seeds
}

\author{
Shuo Wang ${ }^{1,5}$, Gui-Xi Li ${ }^{1,5}$, Cong-Cong Tan ${ }^{1,5}$, Rui He ${ }^{1,5}$, Li-Juan Kang ${ }^{1,5}$, Jun-Tao Lu1, Xiao-Qing Li, ${ }^{1,2}$, \\ Qing-Shan Wang ${ }^{1,2}$, Pei-Fang Liü2,3, Qiong-Li Zhai ${ }^{4} \&$ Yu-Mei Feng ${ }^{1,2}$
}

Bone metastases occur in most advanced breast cancer patients and cause serious skeletalrelated complications. The mechanisms by which bone metastasis seeds develop in primary tumors and specifically colonize the bone remain to be elucidated. Here, we show that forkhead box F2 (FOXF2) functions as a master transcription factor for reprogramming cancer cells into an osteomimetic phenotype by pleiotropic transactivation of the BMP4/ SMAD1 signaling pathway and bone-related genes that are expressed at early stages of bone differentiation. The epithelial-to-osteomimicry transition regulated by FOXF2 confers a tendency on cancer cells to metastasize to bone which leads to osteolytic bone lesions. The BMP antagonist Noggin significantly inhibits FOXF2-driven osteolytic bone metastasis of breast cancer cells. Thus, targeting the FOXF2-BMP/SMAD axis might be a promising therapeutic strategy to manage bone metastasis. The role of FOXF2 in transactivating bone-related genes implies a biological function of FOXF2 in regulating bone development and remodeling.

\footnotetext{
${ }^{1}$ Department of Biochemistry and Molecular Biology, Tianjin Medical University Cancer Institute and Hospital, National Clinical Research Center of Cancer, Tianjin 300060, China. ${ }^{2}$ Key Laboratory of Breast Cancer Prevention and Treatment of the Ministry of Education, Tianjin Medical University Cancer Institute and Hospital, National Clinical Research Center of Cancer, Tianjin 300060, China. ${ }^{3}$ Department of Radiology, Tianjin Medical University Cancer Institute and Hospital, National Clinical Research Center of Cancer, Tianjin 300060, China. ${ }^{4}$ Department of Pathology, Tianjin Medical University Cancer Institute and Hospital, National Clinical Research Center of Cancer, Tianjin 300060, China. ${ }^{5}$ These authors contributed equally: Shuo Wang, Gui-Xi Li, Cong-Cong Tan, Rui He, Li-Juan Kang. Correspondence and requests for materials should be addressed to Y.-M.F. (email: ymfeng@tmu.edu.cn)
} 
B one is the most common site of distant metastasis in breast cancer patients. Bone metastasis occurs in $80 \%$ of patients with advanced breast cancer. Most breast cancer bone metastases generate osteolytic bone lesions associated with a variety of bone complications that seriously affect the life quality of patients, and are associated with a dismal prognosis 1 . The prevention and treatment of bone metastasis remain challenging in the clinic. Elucidating the cellular and molecular mechanisms underlying breast cancer osteotropism could contribute to the development of strategies for predicting and managing bone metastasis. Organ-specific metastasis depends on the intrinsic molecular characteristics of cancer cells, referred to as "preselected" seeds ${ }^{2}$, and a suitable microenvironment in host organs prepared by signals released from primary and/or disseminated cancer cells, referred to as a "pre-metastatic niche" 3 . However, the mechanisms by which primary cancer cells are programmed into bone metastasis seeds and disseminated cancer cells interact with the skeletal microenvironment to flourish in bone and generate osteolytic bone lesions remain to be elucidated.

Breast cancer cells can obtain osteomimetic features to resemble osteocytes by ectopically coexpressing bone-related genes (BRGs) involved in bone and bone-matrix remodeling. Osteomimetic cancer cells possessing bone metastatic capability ${ }^{4}$ preferentially home to the bone microenvironment ${ }^{5}$, wherein they survive ${ }^{6}$ and colonize ${ }^{7,8}$. Our previous gene expression profiling data set ${ }^{9}$ and online data sets ${ }^{10}$ of primary breast cancer tissues indicate that the gene encoding mesenchymal transcription factor (TF) forkhead box F2 (FOXF2) is ectopically coexpressed with BRGs. Among the set of BRGs, multiple genes are candidate transcriptional targets of FOXF2. FOXF2 normally plays critical roles in the maintenance of tissue homeostasis by promoting the differentiation of mesenchymal cells and inhibiting the mesenchymal transformation of adjacent epithelial cells during embryonic development and tissue differentiation ${ }^{11}$. Studies by our group ${ }^{12-15}$ and other groups ${ }^{16-19}$ have demonstrated that the deregulation of FOXF2 expression is involved in the tumorigenesis, progression, and metastasis of breast cancer and other cancer types. FOXF2 deficiency significantly accelerates the visceral metastasis of basal-like breast cancer (BLBC) ${ }^{12}$. However, the role of high ectopic FOXF2 expression in breast cancer cells remains to be explored.

Here, we provide clinical and experimental evidence to illustrate the role of FOXF2 in breast cancer bone metastasis and uncover mechanisms underlying the osteomimetic formation and osteolytic bone metastasis of breast cancer cells, in which FOXF2 programs epithelium-to-osteomimicry transition (EOT) by pleiotropic transactivation of the BMP4/SMAD1 signaling pathway and bone-related genes that are expressed at early stages of bone differentiation. Our findings suggest that targeting the FOXF2-BMP/SMAD axis might be a promising therapeutic strategy to manage breast cancer bone metastasis.

\section{Results}

FOXF2 expression is correlated with bone-specific metastasis. To investigate the role of FOXF2 in breast cancer bone metastasis, we first analyzed the FOXF2 expression pattern in the luminal and triple-negative/basal-like subtypes of breast cancer based on the GSE12777_GSE15026_GSE65194 data set of human breast cancer cell lines and the E-MTAB-365 and GSE3494 data sets of primary breast cancer tissues. The results confirmed our previously published result that FOXF2 mRNA levels were significantly higher in triple-negative/basal-like subtype than in luminal subtype in both cell lines (Fig. 1a) and tissues (Fig. 1b). Then, we analyzed the relationship between FOXF2 expression and organ specificity of metastasis in the luminal and triple-negative subtypes of breast cancer. FOXF2 mRNA levels in primary breast cancer tissues that developed distant metastasis were detected by reverse transcription-quantitative polymerase chain reaction (RT-qPCR). The patients were divided into high FOXF2 mRNA level $\left(F O X F 2_{\text {high }}\right)$ and low FOXF2 mRNA level $\left(F O X F 2_{\text {low }}\right)$ groups, using the optimal cutoff value of FOXF2 mRNA expression for distinguishing bone metastasis-free survival (BMFS) statuses in overall cases and cases stratified by subtypes. Kaplan-Meier survival analysis showed that bone metastasis was a more frequent occurrence in patients in $F O X F 2_{\text {high }}$ group than in those in FOXF2 low group. Stratified analysis based on the hormone receptor status of tumors revealed that $F O X F 2_{\text {high }}$ patients in both luminal and triple-negative breast cancer (TNBC) groups had a shorter BMFS (Fig. 1c). The validation based on the GSE2034_GSE2603 data set confirmed that patients with $F O X F 2_{\text {high }}$ tumors had a shorter BMFS than those with $F O X F 2_{\text {low }}$ tumors among overall cases and among stratified cases of luminal and TNBC subtypes (Fig. 1d). We also analyzed the relationship between FOXF2 expression and distant metastasis-free survival (DMFS) or non-bone/other organ metastasis-free survival (NBMFS) in overall cases and in different subtype cases based on our RT-qPCR data of primary breast cancer tissues. The results showed that FOXF2 mRNA level was positively correlated with DMFS in TNBC subtype and with NBMFS in both luminal and TNBC subtypes (Supplementary Fig. 1). These data indicate that breast cancer with high FOXF2 expression has a propensity to metastasize to bone, which is not affected by hormone receptor status.

FOXF2 drives bone-specific breast cancer metastasis. To investigate the role of FOXF2 in regulating breast cancer bone metastasis, we established 4T1 cells stably overexpressing Foxf2-Flag fusion protein (4T1-Foxf2) and their controls (4T1Vector) via lentiviral infection (Fig. 2a) for mouse xenograft experiments. The cells were orthotopically injected into the fat pad of female nude mice. The primary tumors formed by 4T1Foxf2 cells were larger than those formed by 4T1-Vector cells (Fig. 2b), and there was more angiogenesis in 4T1-Vector tumors than in 4T1-Foxf2 tumors (Fig. 2c). X-ray images of hind leg bones showed that 4T1-Foxf2 tumors led to serious osteolytic bone lesions, which were validated by hematoxylin and eosin (H\&E) and tartrate-resistant acid phosphatase (TRAP) staining, whereas mice-bearing 4T1-Vector tumors had significantly fewer osteolytic bone lesions (Fig. 2d). The metastatic 4T1-Foxf2 cells isolated from the bone marrow and cultured in vitro formed more colonies, and the colonies were larger than those formed by metastatic 4T1-Vector cells (Fig. 2e). However, Foxf2 overexpression significantly suppressed lung metastases that were observable on the lung surfaces and identified by histological examination (Fig. 2f). Liver metastases were also suppressed by Foxf2 overexpression and identified by histological examination in the livers of mice-bearing 4T1-Vector tumors only (Fig. 2g). We further validated the role of Foxf2 in regulating breast cancer metastasis using a $4 \mathrm{~T} 1 / \mathrm{BALB} / \mathrm{c}$ orthotopic xenograft mouse models with Foxf2 loss- and gain-of-function (Supplementary Fig. $2 \mathrm{a}-\mathrm{e}$ ). The results confirmed the role of FOXF2 in promoting bone metastasis and suppressing lung and liver metastases. The role of FOXF2 in suppressing the visceral metastasis of BLBC is consistent with the findings in our previous report ${ }^{12}$.

FOXF2 enhances bone-specific metastatic potential. To investigate the role of FOXF2 in regulating various processes underlying breast cancer bone metastasis, we forced the ectopic expression of FOXF2 in MCF-7 cells and overexpressed or knocked down FOXF2 in MDA-MB-231 cells. The cancer cells 
a

GSE12777_15026_65194 cell lines dataset

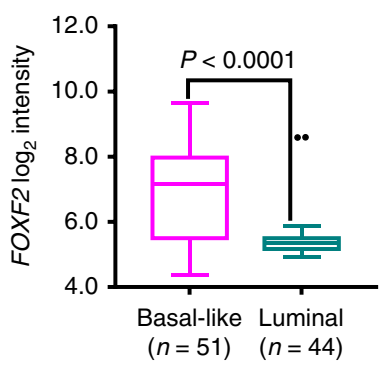

C

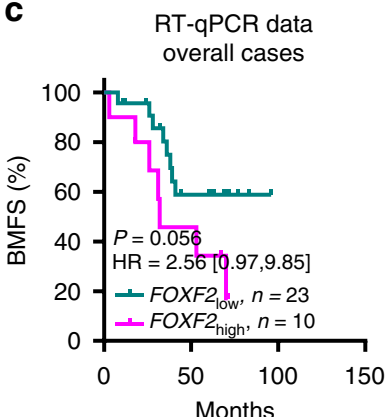

d

GSE2034_GSE2603 data overall cases

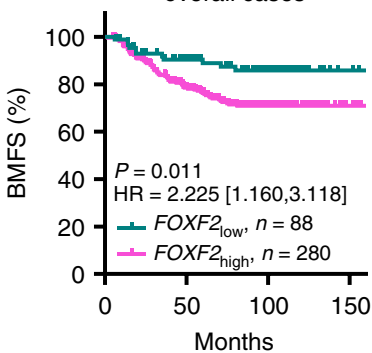

b
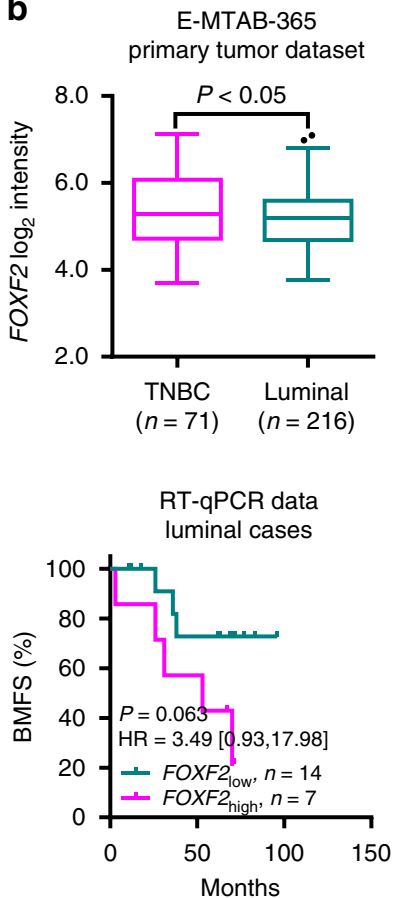

GSE2034_GSE2603 data luminal cases

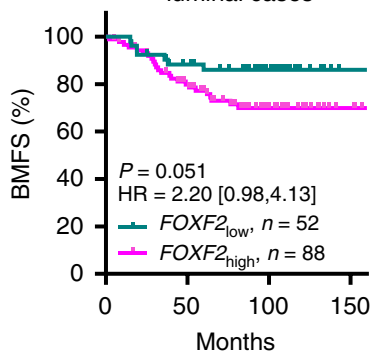

GSE3494

primary tumor dataset
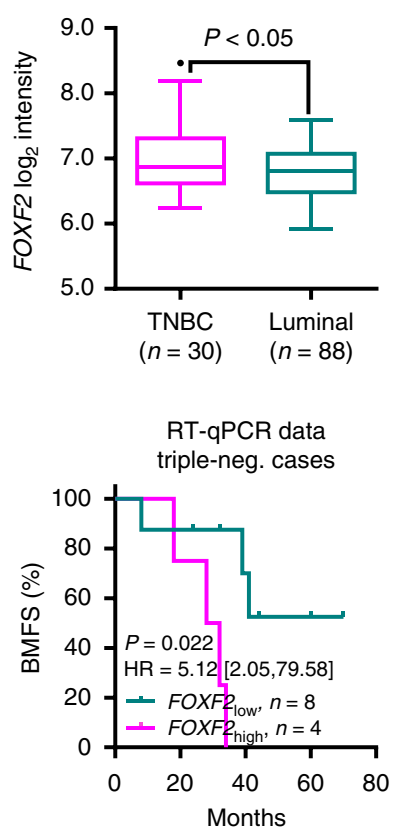

GSE2034_GSE2603 data triple-neg. cases

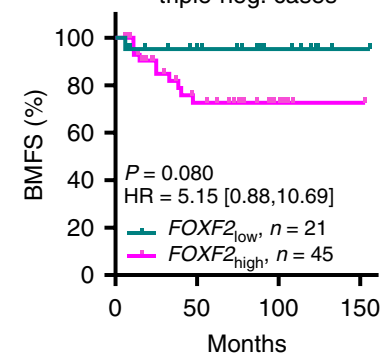

Fig. 1 Breast cancers with high FOXF2 expression have a propensity to metastasize to bone. a-b FOXF2 expression levels in the luminal and basal-like/ triple-negative subtypes of human breast cancer were compared by chi-square tests. FOXF2 mRNA levels were mined from the GSE12777_GSE15026_GSE65194 data set of breast cancer cell lines $(n=95)$; a and the E-MTAB-365 $(n=287)$ and GSE3494 ( $n=118)$ data sets of primary breast cancer tissues (b). The boxplots show the minimum, 25 percentile, median, 75 percentile, maximum, and outliers. c FOXF2 mRNA levels in primary breast cancer tissues that developed distant metastasis $(n=33)$ were detected by RT-qPCR. $\mathbf{d}$ FOXF2 mRNA levels in primary breast cancer tissues ( $n=368$ ) were mined from the GSE2034_GSE2603 data set. The Kaplan-Meier survival curve shows the BMFS status of patients in the FOXF2 and FOXF2 low groups. The overall, luminal subtype and TNBC subtype cases were analyzed separately

with altered FOXF2 expression were evaluated in vitro for chemotactic migration, heterogeneous cell-cell adhesion, and soft agar colony formation in the MC3T3E1 cell-mimic bone microenvironment and BEAS-2B cell-mimic lung microenvironment. The results revealed that the chemotactic migration of MCF-7 and MDA-MB-231 cells toward MC3T3E1 cells (Fig. 3a), heterogeneity adhesion to MC3T3E1 cells (Fig. 3b), and anchorageindependent growth in soft agar with conditioned medium (CM) from MC3T3E1 (Fig. 3c) were significantly increased by forced expression of FOXF2 and decreased by knockdown of FOXF2. In contrast, these properties of TNBC/BLBC MDA-MB-231 cells were suppressed by FOXF2 overexpression and increased by FOXF2 knockdown in the BEAS-2B cell-mimic lung microenvironment. However, forced ectopic expression of FOXF2 did not affect these capabilities of luminal breast cancer MCF-7 cells in the BEAS-2B cell-mimic lung microenvironment (Fig. 3a-c). Since pulmonary fibroblasts and hepatic stellate cells are the most abundant stromal cell types in the lung and liver, primary human pulmonary fibroblasts (HPFs) and human hepatic stellate cells
(HHSCs) were also used to mimic the lung and liver microenvironment to evaluate the lung and liver metastatic potential of the above cancer cells. We observed that FOXF2 negatively regulated the chemotactic migration, heterogeneous cell-cell adhesion, and soft agar colony formation of MDA-MB-231 cells in the HPF-mimic lung microenvironment and HHSC-mimic liver microenvironment. Surprisingly, forced ectopic expression of FOXF2 enhanced these capabilities of MCF-7 cells in the HPFmimic lung microenvironment and HHSC-mimic liver microenvironment (Supplementary Fig. 3a-c).

Because the bone metastasis of breast cancer cells results in osteolytic lesions, we tested whether FOXF2 drives cancer cells to induce osteoclastogenesis. Pre-induced primary preosteoclasts were incubated with the CM from cancer cells treated as above. TRAP staining showed that the number and size of TRAP+ osteoclasts induced by the CM from FOXF2-overexpressing cancer cells were significantly increased compared with those induced by control cell CM. Conversely, the number and size of TRAP+ osteoclasts were reduced by the CM from 


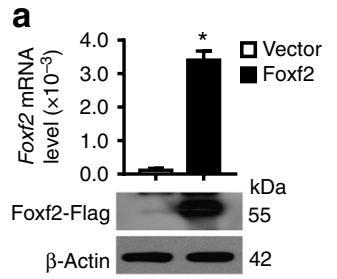

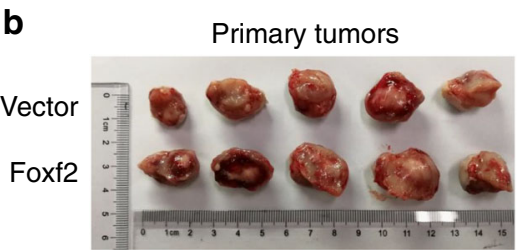

Day 30 after fat pad injection

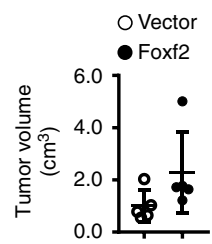

e

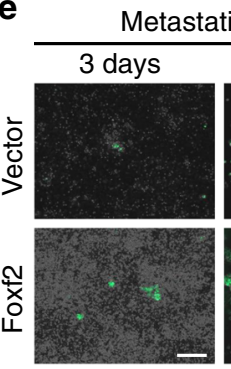

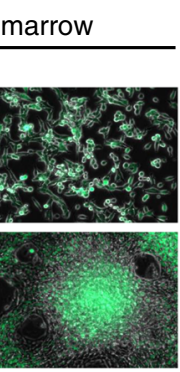

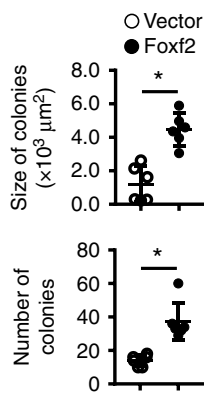

d

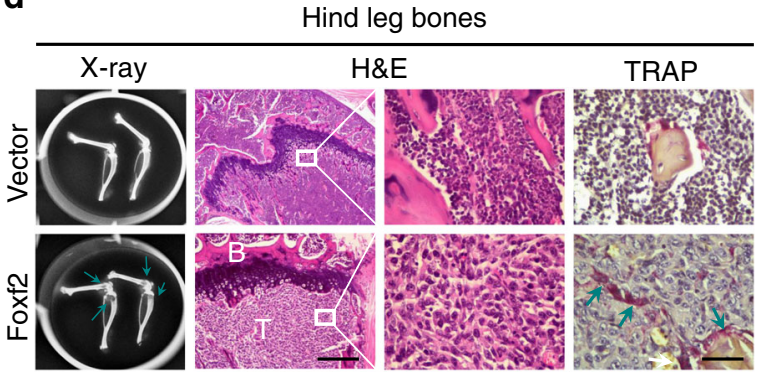

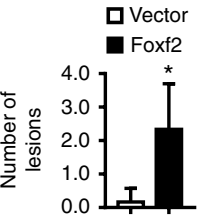

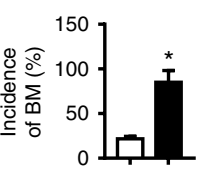

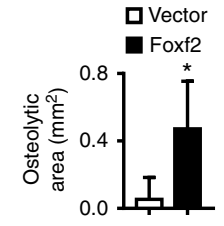

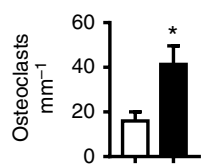

$\mathbf{f}$

Lung
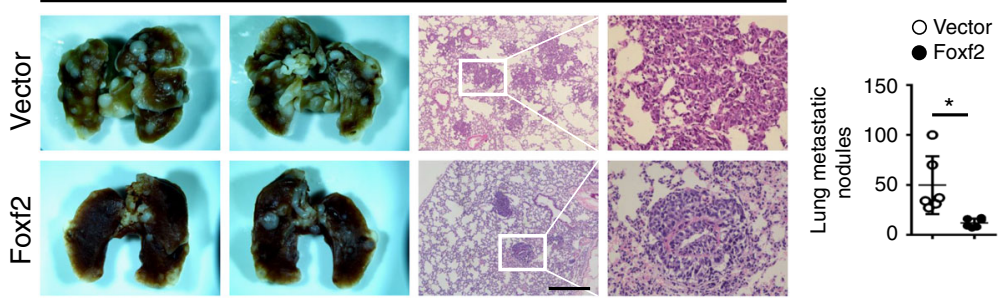

g

Liver
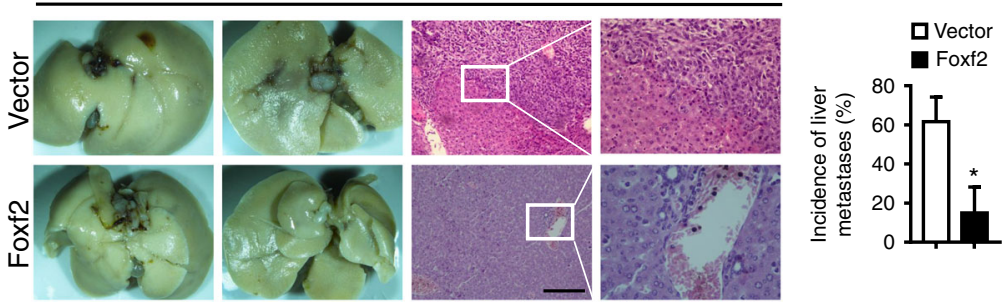

Fig. 2 Foxf2 promotes the bone-specific metastasis of breast cancer cells in vivo. A total of $2 \times 10^{5} 4 \mathrm{~T} 1$ cells infected with LV-Foxf2-EGFP (Foxf2) or LVEGFP vector (Vector) were injected into the fat pad of female nude mice ( $n=5$ per group). The mice were killed by cervical dislocation on day 30 after cell injection. a Foxf2 mRNA and protein levels in the indicated cells were detected by RT-qPCR and immunoblot. $\mathbf{b}$ The volume of xenograft tumor volume was calculated. c Histological evaluation of xenograft tumors was identified after H\&E staining. Scale bars: $100 \mu \mathrm{m}$. $\mathbf{d}$ The osteolytic lesions and metastases in hind leg bones were observed by X-ray, H\&E staining, and TRAP staining. The number of osteolytic lesions, osteolytic area, and log 2 signal intensity in hindleg bones detected by X-ray were calculated. Scale bars: $500 \mu \mathrm{m}$ for H\&E staining and $50 \mu \mathrm{m}$ for TRAP staining. Arrows point to osteolytic lesions or TRAP + cells. e The metastatic cells in the bone marrow of the hind legs were collected and cultured in vitro for 3 or 10 days. The size and the number of metastatic cell colonies were determined under a fluorescence microscope and statistically analyzed. Scale bars, $200 \mu \mathrm{m}$. $\mathbf{f}-\mathbf{g}$ Metastatic nodules on two sides of the lung (f) and liver ( $\mathbf{g}$ ) surface were photographed, identified by H\&E staining and statistically analyzed. The metastatic nodules were counted, or the incidence of metastasis was calculated. Scale bars: $200 \mu \mathrm{m} .{ }^{\star} P<0.05$ by Student's $t$ test. Error bars are defined as s.d. 
a

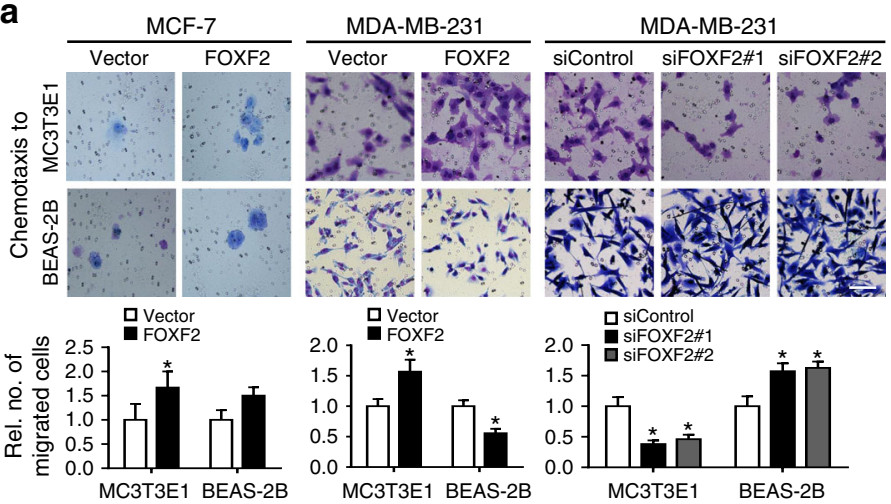

b

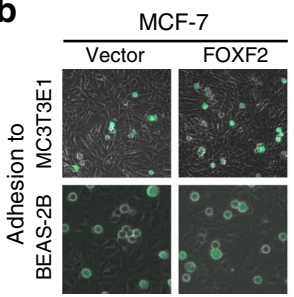

MDA-MB-231
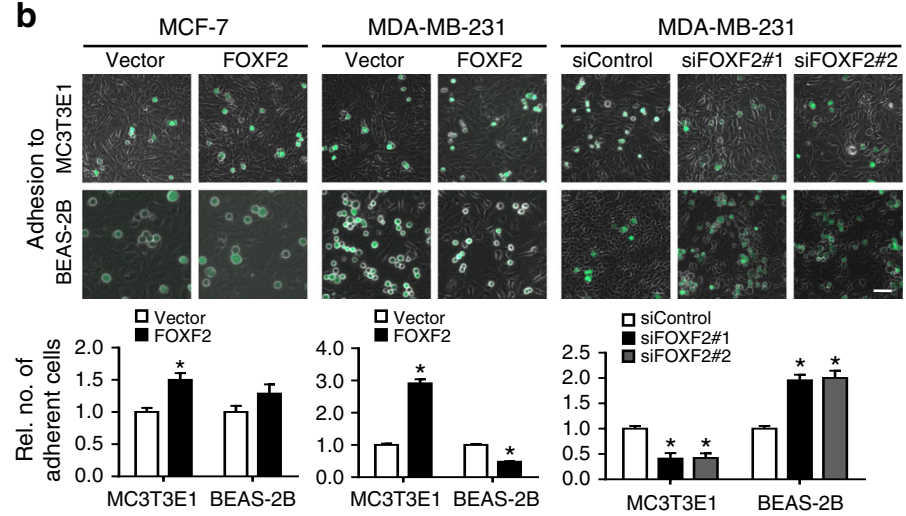

C
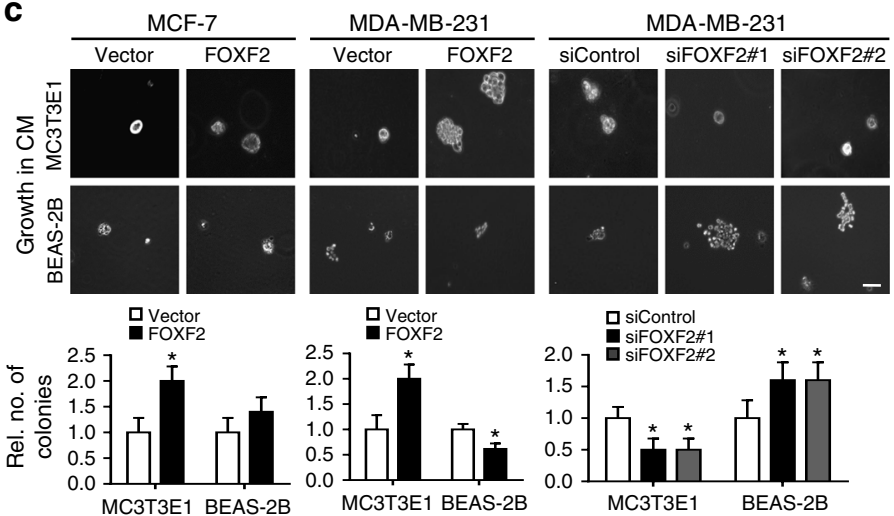

d
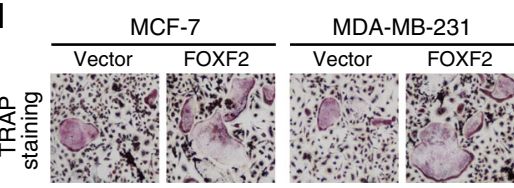

MDA-MB-231
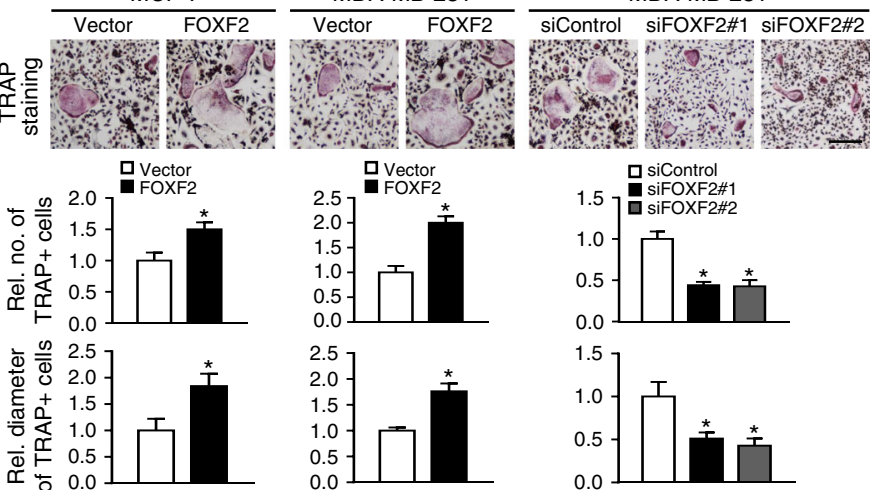

FOXF2-depleted cells compared with that from control cells (Fig. 3d).

Together, these results indicate that high FOXF2 expression confers the advantages of homing to, residing in, and growing in the bone microenvironment on both luminal and BLBC cells, which promotes osteoclast formation and subsequently results in bone lesions. However, FOXF2 may play opposing roles in luminal and BLBC cells for regulating the lung and liver metastatic potential. FOXF2 controls the colonization of BLBC cells in the lung and liver microenvironment, and FOXF2 
Fig. 3 FOXF2 enhances the bone-specific metastasis potential of breast cancer cells. MCF-7 and MDA-MB-231 cells were treated as indicated. a Chemotactic migration of cancer cells toward MC3T3E1 or BEAS-2B cells was assessed by transwell assays. $\mathbf{b}$ Adhesion of cancer cells to MC3T3E1 or BEAS-2B cells was assessed by adding cancer cells to MC3T3E1 or BEAS-2B cells at $100 \%$ saturation, followed by incubation for 30 min. c Anchorageindependent growth of cancer cells in the CM from MC3T3E1 or BEAS-2B cells was assessed by soft agar colony-formation assays. $\mathbf{d}$ The ability of cancer cells to induce osteoclast differentiation was assessed by incubation of pre-induced primary preosteoclasts with cancer cell $\mathrm{CM}$ containing $50 \mathrm{ng} \mathrm{ml}^{-1}$ RANKL. The number and size of mature osteoclasts with TRAP-positive multinucleated ( $\geq 3$ nuclei) were analyzed. Scale bars: $100 \mu \mathrm{m}$ for (a-c) and $500 \mu \mathrm{m}$ for $(\mathbf{d}) .{ }^{\star} P<0.05$ by Student's $t$ test. Error bars are defined as s.d.

depletion enables BLBC cells to colonize the lung and liver. FOXF2 may enhance this potential of luminal cells, which is facilitated by stromal cells in the lung and liver.

FOXF2 pleiotropically transactivates BRGs. To investigate the mechanism by which FOXF2 drives breast cancer bone metastasis, we screened genes that were coexpressed with FOXF2 based on our gene expression profiling data set of 49 primary breast cancer tissues 9 and the Gene expression-based Outcome for Breast cancer Online (GOBO, http://co.bmc.lu.se/gobo/) tool ${ }^{10}$. The cutoff value of Pearson's correlation coefficient was defined as 0.3 , and $P$-value was set to less than 0.05 . A set of BRGs was identified as coexpression with FOXF2 in breast cancer tissues (Fig. 4a; Supplementary Fig. 4a). The validation based on the GSE20685 data set confirmed that FOXF2 expression positively correlated with the expression of BRGs, e.g., runt-related transcription factor 2 (RUNX2), periostin (POSTN), cathepsin $\mathrm{K}$ (CTSK), and lysyl oxidase-like 2 (LOXL2; Fig. 4b). These data indicate that FOXF2 is involved in the formation of the osteomimetic phenotype of breast cancer cells.

Since breast cancer cells with osteomimetic features have the advantage of osteotropism, we analyzed the relationship between the expression of FOXF2 and BRGs in primary breast cancer tissues with bone-only metastasis based on the GSE2034_GSE2603 data set. The heatmap (Fig. 4c) shows that patients with high expression of FOXF2 or BRGs in tumors had a higher frequency $(47 / 181$ or $34 / 115)$ of suffering bone-only metastasis than those with low expression of FOXF2 or BRGs in tumors (14/126 or 27/192; Fig. 4d), indicating that breast cancers with high expression of FOXF2 or coexpression of FOXF2 and BRGs preferentially metastasize to bone.

To investigate whether FOXF2 directly regulates the set of BRGs, we analyzed the $2-\mathrm{kb}$ upstream region of the promoters for the FOXF2-binding sequence. Indeed, multiple BRGs are potentially regulated by FOXF2 (Table 1). Chromatin immunoprecipitation (ChIP) assays confirmed the binding of FOXF2 to the promoter regions of BRGs containing the candidate-binding sites in MCF-7 and/or MDA-MB-231 cells (Supplementary Fig. $4 \mathrm{~b}$ ). The detection of proteins encoded by BRGs in MCF-7 and MDA-MB-231 cells with FOXF2 overexpression and/or FOXF2 knockdown showed that FOXF2 positively regulated the protein expression of cadherin 11 (CDH11) and spondin 1 (SPON1) in both MCF-7 and MDA-MB-231 cells, as well as the protein expression of integrin subunit beta-like 1 (ITGBL1), POSTN/osteoblast-specific factor (OSF-2), secreted protein acidic and cysteine-rich (SPARC)/osteonectin (OSN), and RUNX2 in only MDA-MB-231 cells (Fig. 4e).

Although FOXF2 was coexpressed with a set of BRGs in breast cancer, FOXF2 directly regulated some BRGs, but not others. We speculated that FOXF2 may regulate the bone morphogenetic protein (BMP)/SMAD signaling pathway that is crucial for bone differentiation and remodeling. The detection of BMPs and SMADs expression in the above cells showed that FOXF2 positively regulated BMP4, SMAD1 and phosphorylated SMAD1 (p-SMAD1), negatively regulated p-SMAD2 and p-SMAD3, and did not regulate BMP2, SMAD2, SMAD3, SMAD4, SMAD5, SMAD6, SMAD7, or SMAD9 expression (Fig. 5a). The detection of secreted proteins in the CM confirmed that FOXF2 positively regulated the secretion of BMP4 but not of BMP2 (Supplementary Fig. 5). Since BMP4 and SMAD1 are candidate targets of FOXF2 (Fig. 5b), we investigated the direct effect of FOXF2 on BMP4 and SMAD1. ChIP assays showed that FOXF2 protein bound to the BMP4 and SMAD1 promoter regions containing putative FOXF2-binding sequences in both MDA-MB-231 and MCF-7 cells (Fig. 5c). Dual-luciferase reporter assays validated that FOXF2 positively regulated the activation of the BMP4 and SMAD1 promoters (Fig. 5d), as well as the BMP reporter (Fig. 5e) in MDA-MB-231 and MCF-7 cells. These results suggest that FOXF2 directly transactivates the expression of BMP4 and SMAD1, implying that FOXF2 programs osteomimetic phenotype in breast cancer cells by pleiotropic transactivation of BRGs and the BMP/SMAD signaling pathway.

To provide clinical evidence of the role of the FOXF2/BMP/ $\mathrm{SMAD}$ axis in breast cancer bone metastasis, we analyzed the expression of BMP4 and SMAD1 in primary breast cancer tissues that developed bone-only metastasis $(n=61)$ or metastases in other distant organs with or without bone metastases $(n=36)$ based on the GSE2034_GSE2603 data set; we also analyzed the expression of these genes in metastatic tissues in bone $(n=18)$ and in other distant organs $(n=47)$ based on the GSE14020 data set. The results showed that BMP4 was highly expressed in primary cancers that developed bone-only metastasis and metastatic cancers in bone, compared with primary cancers that developed other distant organ metastasis and metastatic cancers in other organs, respectively. SMAD1 was highly expressed in metastatic cancers in bone, but not in primary tumors that developed bone-only metastasis (Fig. $5 \mathrm{f}, \mathrm{g}$ ).

We further analyzed the relevance of BMP4 or SMAD1 mRNA levels in primary breast cancer with bone-specific metastasis based on the GSE2034_GSE2603 data set. All cases were grouped into high and low BMP4 expression groups $\left(B M P 4_{\text {high }}\right.$ and $B M P 4_{\text {low }}$ ) or high and low $S M A D 1$ expression groups ( $S M A D 1_{\text {high }}$ and $S M A D 1_{\text {low }}$ ) according to the optimized cutoff values, which had high sensitivity and specificity in separating all patients into distinct DMFS statuses. The results revealed that the rates of bone-only metastasis-free survival (BOMFS) in $B M P 4_{\text {high }}$ and $S M A D 1_{\text {high }}$ patients were lower than that in $B M P 4_{\text {low }}$ and $S M A D 1_{\text {low }}$ patients (Fig. $5 \mathrm{~h}$ ).

We then combined FOXF2 expression levels with BMP4 or $S M A D 1$ expression levels. The patients were grouped into subgroups of $F O X F 2_{\text {high }} / B M P 4_{\text {high }}, F O X F 2_{\text {high }} / B M P 4_{\text {low }}, F O X-$ $F 2_{\text {low }} / B M P 4_{\text {high }}$, and $F O X F 2_{\text {low }} / B M P 4_{\text {low }}$, or subgroups of $F O X F 2_{\text {high }} / S M A D 1_{\text {high }}, \quad F O X F 2_{\text {high }} / S M A D 1_{\text {low }}, \quad F O X F 2_{\text {low }} /$ $S M A D 1_{\text {high }}$, and $F O X F 2_{\text {low }} / S M A D 1_{\text {low }}$. Among these subgroups, $F O X F 2_{\text {high }} / B M P 4_{\text {high }}$ or $F O X F 2_{\text {high }} / S M A D 1_{\text {high }}$ subgroup had significantly poorer BOMFS than the other subgroups (Fig. 5i), indicating that $B M P 4_{\text {high }}$ and $S M A D 1_{\text {high }}$ contribute to the $F O X F 2_{\text {high }}$-driven bone-specific metastasis of breast cancer. Together, these clinical data support the role of the FOXF2/ $\mathrm{BMP} / \mathrm{SMAD}$ axis in breast cancer bone metastasis. 
a

Our data

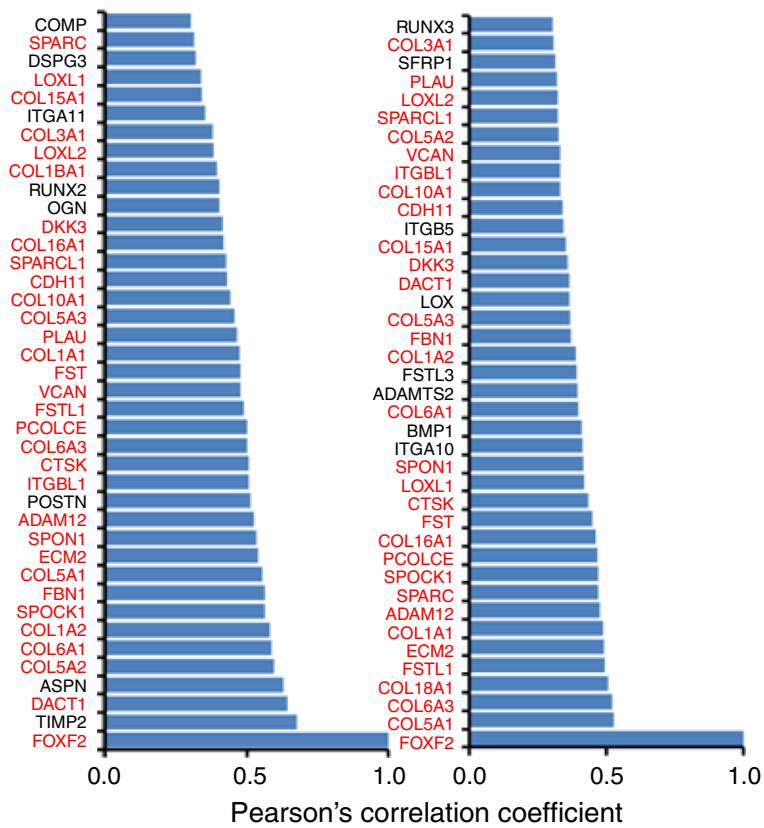

C

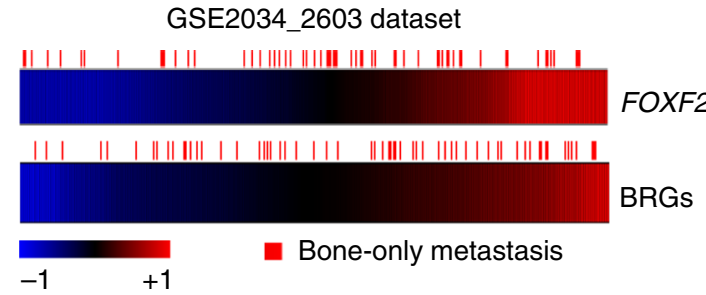

d

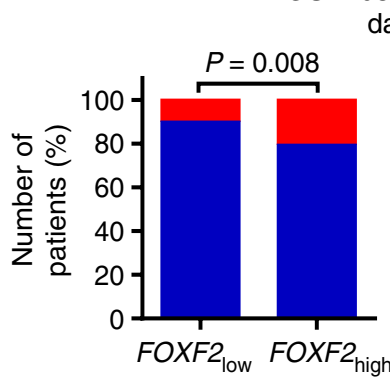

GOBO data
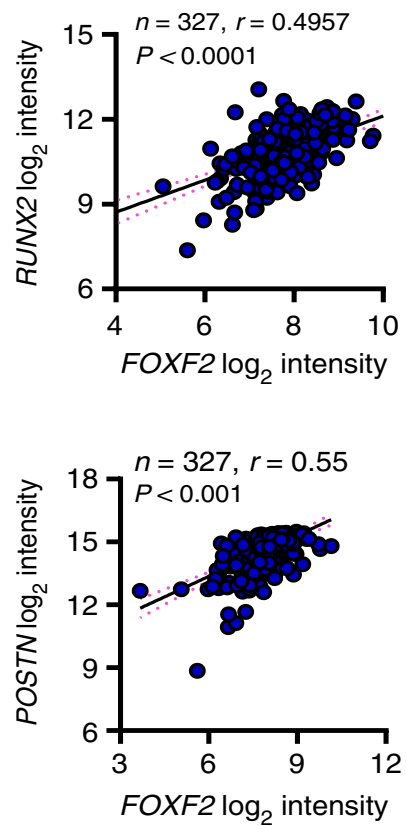

GSE20685 dataset
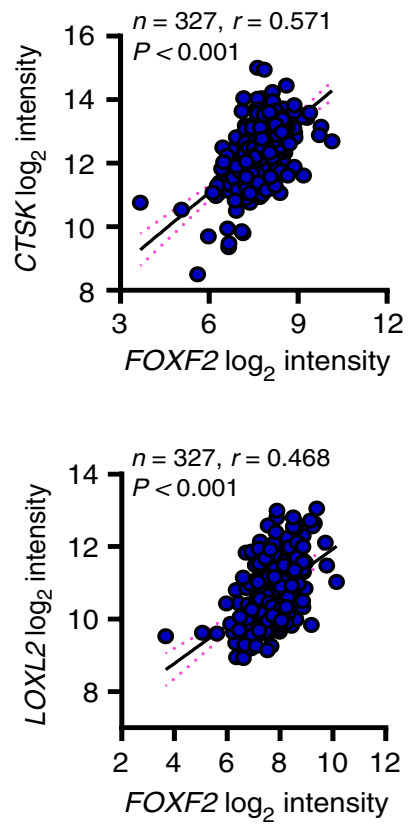

e

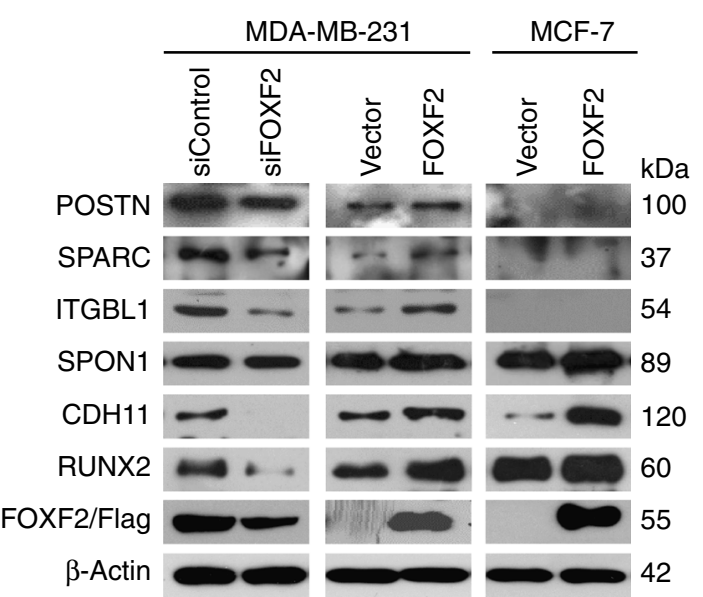

Fig. 4 FOXF2 confers osteomimetic features on breast cancer cells and facilitates bone metastasis. a Genes coexpressed with FOXF2 in breast cancer were analyzed based on our gene expression profiling data set of 49 primary breast cancer tissues and the GOBO tool. The cutoff value of Pearson's correlation coefficient was defined as 0.3. b The correlation of the expression of FOXF2 with BRGs in primary breast cancer tissues based on GSE20685 data set was analyzed by Pearson's correlation analysis. c Heatmaps (top) show the correlation of the frequency of bone-only metastasis (BOM) with the expression of FOXF2 and the set of BRGs coexpressed with FOXF2 based on the GSE2034_GSE2603 data set. The score of the set of BRGs was defined as an average of

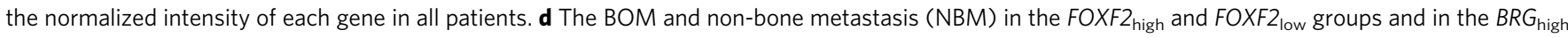
and $B R G_{\text {low }}$ groups were compared. e The expression of proteins encoded by BRGs in the indicated cells was detected by immunoblot

The BMP pathway mediates FOXF2-drived bone-specific metastasis. To further investigate whether the BMP/SMAD signaling pathway mediates the FOXF2-regulated osteotropism of breast cancer metastasis, we added the BMP antagonist Noggin to the culture medium of MCF-7 or MDA-MB-231 cells transfected with FOXF2-expression plasmid or vector control, and added BMP4 to the culture medium of MDA-MB-231 cells transfected with small interfering RNAs (siRNAs) targeting FOXF2 (siFOXF2) or non-targeting siRNA (siControl). Then, we assessed chemotactic migration, heterogeneous cell-cell adhesion, and soft agar colony formation of the above cancer cells in the MC3T3E1 cell-mimic bone microenvironment and the BEAS-2B cell-mimic lung microenvironment. The results revealed that Noggin attenuated FOXF2 overexpression-induced increase in the chemotactic migration of both MCF-7 and MDA-MB-231 cells toward MC3T3E1 cells (Fig. 6a), heterogeneous adhesion to MC3T3E1 cells (Fig. 6b), anchorageindependent growth in soft agar (Fig. 6c) containing MC3T3E1 $\mathrm{CM}$, and osteoclast formation (Fig. 6d). Consistently, BMP4 restored the FOXF2 depletion-caused suppression of these 
Table 1 BRGs potentially targeted by FOXF2

\begin{tabular}{llll} 
Gene symbol & Reference sequence number & Gene description & FOXF2-binding site related to TSS \\
\hline ITGBL1 & NM_001271756.1 & Integrin, beta-like 1 isoform 3 & $-659 /-652$ \\
CSPG2 & NM_001126336.2 & Chondroitin sulfate proteoglycan 2/versican (VCAN) & $-1199 /-1192$ \\
COMP & NM_000095.2 & Cartilage oligomeric matrix protein & $-1422 /-1415$ \\
DSPG3 & NM_004950.4 & Dermatan sulphate proteoglycan 3/epiphycan (EPYC) & $-32 /-25$ \\
& & & $-547 /-540$ \\
& & Follistatin & $-1585 /-1972 /-1965$ \\
FST & NM_006350.3 & Osteoglycin & $-1050 /-1043$ \\
OGN & NM_014057.4 & Periostin/osteoblast-specific factor 2 (OSF-2) & $-154 /-147$ \\
POSTN & NM_001135934.1 & Secreted protein, acidic, cysteine-rich/osteonectin (OSN) & $-990 /-983$ \\
SPARC & NM_001309443.1 & Spondin 1 & $-795 /-788$ \\
SPON1 & NM_006108.3 & Cathepsin K & $-69 /-62$ \\
CTSK & NM_000396.3 & & $-586 /-579$ \\
& & Lysyl oxidase-like 2 & $-732 /-725$ \\
LOXL2 & NM_002318.2 & Plasminogen activator/urokinase (uPA) & $-1602 /-1595$ \\
PLAU & NM_001145031.2 & & $-413 /-406$ \\
& & & $-1082 /-1075$ \\
& & & $-1109 /-1102$ \\
\hline
\end{tabular}

capacities of MDA-MB-231 cells (Fig. 6a-d). However, Noggin and BMP4 did not significantly affect the FOXF2 alterationleaded changes in these capacities of MCF-7 and MDA-MB-231 cells in the BEAS-2B cell-mimic lung microenvironment (Fig. 6a-c), HPF-mimic lung microenvironment or HHSCmimic liver microenvironment (Supplementary Fig. 6a-c). These results indicate that the BMP/SMAD signaling pathway mediates the FOXF2-regulated osteotropism of breast cancer metastasis and osteoclastogenesis, as well as the metastatic potential of luminal cells toward the lung and liver, but is not remarkably involved in FOXF2 deficiency-conferred the lungand liver-specific metastatic potential on BLBC cells.

To further verify the role of the BMP/SMAD signaling pathway in mediating the promotion of breast cancer bone-specific metastasis by FOXF2, we established stable MDA-MB-231 cells with FOXF2 overexpression (231-FOXF2) or vector control (231Vector) via lentiviral infection for mouse xenograft experiments by ventricle injection. Consistent with the results generated from the fat pad injection of 4T1-Foxf2 cells (Fig. 2), FOXF2 overexpression significantly enhanced osteolytic bone metastases in limbs, as shown by bioluminescence photography (Fig. 7a) and molybdenum target X-ray photography (Fig. 7b) and validated by $\mathrm{H} \& \mathrm{E}$ and TRAP staining (Fig. 7c), but significantly suppressed liver metastases (Fig. 7d). As expected, the BMP antagonist Noggin significantly attenuated FOXF2-promoted bone metastasis (Fig. 7a-c), but did not affect FOXF2-suppressed visceral metastasis (Fig. 7d). The fat pad injection of 4T1-Foxf2 cells confirmed that inhibition of the BMP/SMAD signaling pathway by Noggin significantly reduced FOXF2-driven bone metastasis (Fig. 7e), but did not affect the role of FOXF2 in suppressing visceral metastasis (Fig. 7f). We also performed FOXF2 knockdown and BMP4 stimulation experiments using 231-Luc-BM cells. As respected, the results showed that FOXF2 deficiency decreased bone metastasis, and that BMP4 treatment restored bone metastases suppressed by FOXF2 depletion (Supplementary Fig. $7 \mathrm{a}-\mathrm{c}$ ). Further immunohistochemistry staining for $\mathrm{pSmad} 1 / 5$ in the primary tumors of $4 \mathrm{~T} 1 / \mathrm{BALB} / \mathrm{c}$ mouse model confirmed that Foxf 2 overexpression increased $\mathrm{pSmad} 1 / 5$ expression, and this effect was attenuated by Noggin treatment (Supplementary Fig. 7d). These results indicate that FOXF2 promotes breast cancer bone metastasis by directly increasing and activating the $\mathrm{BMP} / \mathrm{SMAD}$ signaling pathway.
CTSK mediates FOXF2-induced osteoclastogenesis. CTSK is a well-known inducer of osteoclasts and a marker of activated osteoclasts. Because CTSK is coexpressed with FOXF2 (Fig. 4a, b) and the CTSK proximal promoter region contains three putative FOXF2-binding sequences, we speculated that FOXF2 may enable breast cancer cells to induce osteoclast maturation by increasing CTSK. ChIP-PCR assays showed that FOXF2 bound to the CTSK proximal promoter region at $-586 /-579 \mathrm{bp}$ and $-732 /-725 \mathrm{bp}$ relative to the transcription start site (TSS) in MDA-MB-231 cells transfected with pcDNA3.1-FOXF2-FLAG (Fig. 8a). Dualluciferase reporter assays revealed that FOXF2 knockdown significantly reduced the luciferase activity in MDA-MB-231 cells transfected with pGL3-CTSK containing the $-732 /-725 \mathrm{bp}$ FOXF2-binding site compared with that in control cells (Fig. 8b). The levels of CTSK mRNA, intracellular protein (Fig. 8c), and secretion (Fig. 8d) were consistently regulated by FOXF2. CTSK protein expression was also increased in bone metastatic tissues formed by MDA-MB-231 cells, especially in cancer cells adjacent to osteolytic bone lesions (Fig. 8e). These results demonstrate that FOXF2 activates the transcription and expression of CTSK by binding to its promoter region in breast cancer cells.

To further investigate the role of the FOXF2-CTSK axis in the interaction of breast cancer cells with bone, we performed bonematrix invasion assays to test whether FOXF2-regulated CTSK secretion by cancer cells led to the degradation and invasion of the bone matrix. MDA-MB-231 cells with FOXF2 knockdown or overexpression, FOXF2 knockdown plus adding CTSK or FOXF2 overexpression plus CTSK knockdown were seeded on transwell inserts pre-coated with bone matrix. We observed that the ability of cancer cells to invade the bone matrix was positively regulated by FOXF2 expression. The addition of CTSK reversed the suppression of bone-matrix invasion by FOXF2 knockdown, and CTSK knockdown abolished the increase in invasion due to FOXF2 overexpression (Fig. 8f). We further tested whether FOXF2-regulated CTSK secretion by cancer cells induce osteoclastogenesis. We found that the addition of CTSK to CM from FOXF2-depleted cancer cells restored the reductions in the number and size of TRAP+ osteoclasts. Conversely, CTSK knockdown attenuated the number and size of TRAP+ osteoclasts induced by the CM from FOXF2-overexpressing cancer cells (Fig. 8g). These data suggest that CTSK mediates the FOXF2-enhanced potential of osteolytic bone metastasis. 
a

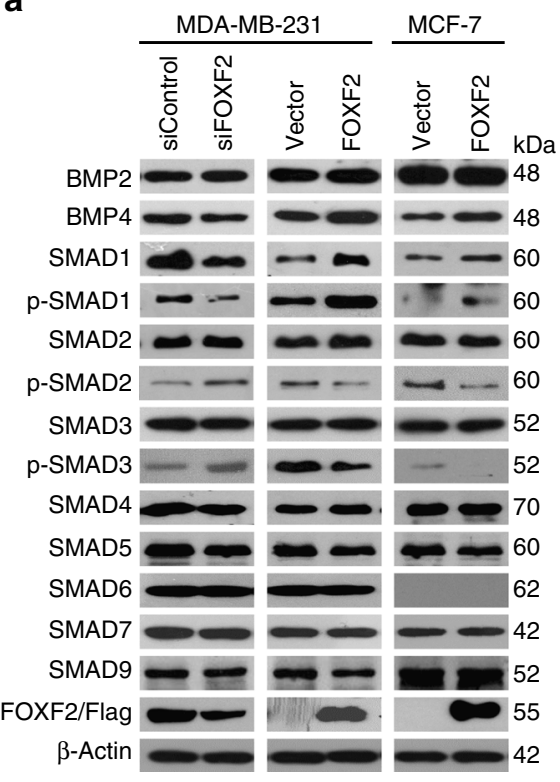

b

\begin{tabular}{lll}
\multicolumn{3}{c}{ Candidate FOXF2 targeted genes } \\
\hline Genes & $\begin{array}{l}\text { Location } \\
\text { related to TSS }\end{array}$ & $\begin{array}{l}\text { Putative FOXF2 } \\
\text { binding sequence }\end{array}$ \\
\hline BMP4 & $-779 /-772$ & AATCAACA \\
SMAD1 & $-322 /-315$ & AATAAACA \\
\hline
\end{tabular}

d

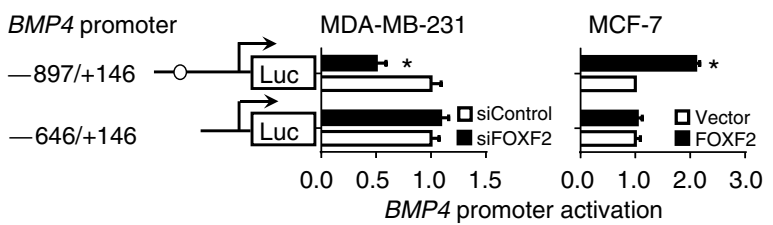

SMAD1 promoter

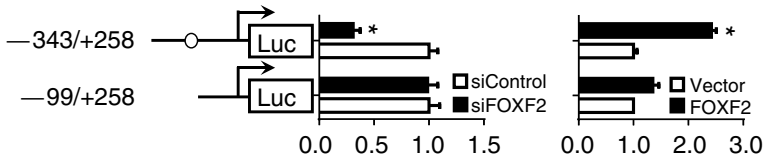

o Putative FOXF2 binding site SMAD1 promoter activation
C

MDA-231/ MCF-7/ FOXF2-Flag FOXF2-Flag

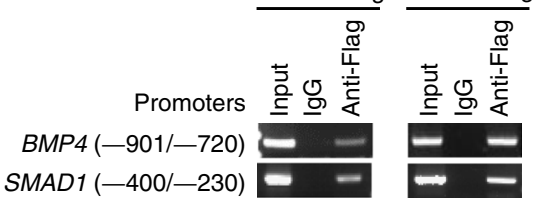

e

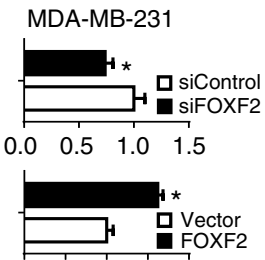

0.00 .51 .01 .52 .0

MCF-7

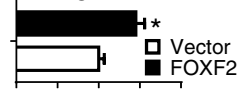

0.00 .51 .01 .52 .0

BMP reporter activation $\mathbf{f}$

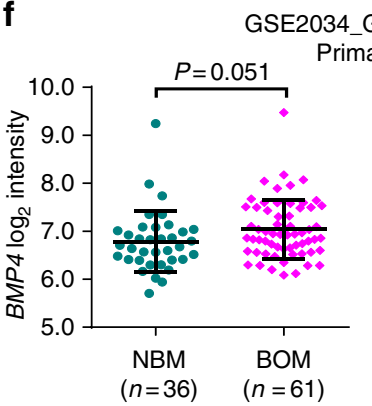

PSE2603 dataset

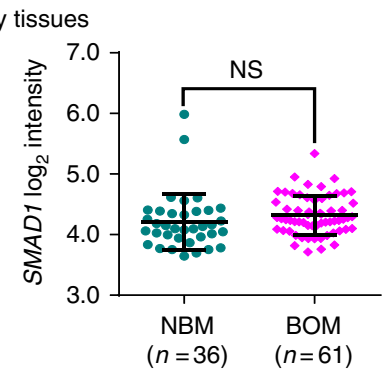

g

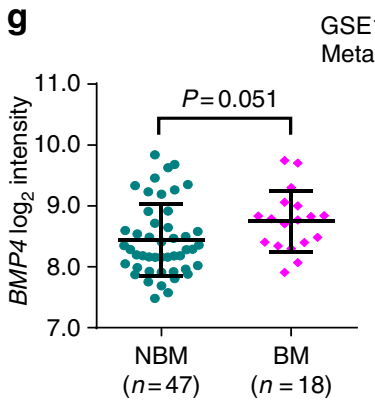

GSE14020 dataset Metastatic tissues h

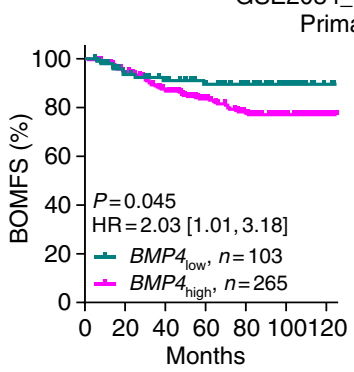

2034 GSE2603 datase

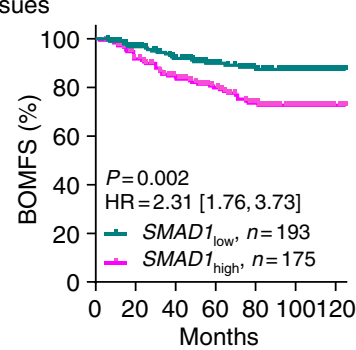

i

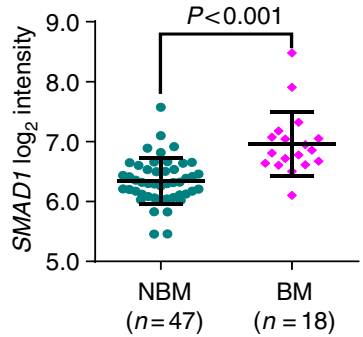

GSE2034_GSE2603 dataset Primary tissues
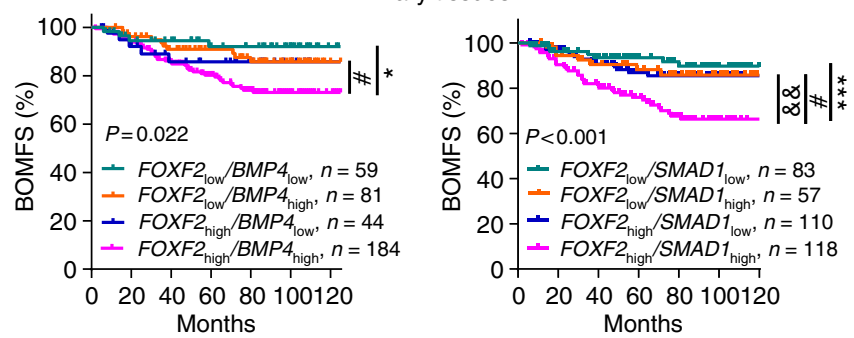

Fig. 5 FOXF2 confers osteomimetic features and osteotropism on breast cancer cells by direct transactivation of the BMP4/SMAD1 signaling pathway. a The protein levels of BMPs and SMADs in the indicated cells were detected by immunoblot. $\mathbf{b}$ Candidate FOXF2 target genes in the BMP/SMAD signaling pathway are shown. $\mathbf{c}$ The binding of FOXF2 to the BMP4 and SMAD1 promoters containing or lacking FOXF2-binding sequences was assessed by ChIP assays. $\mathbf{d}$ BMP4 and SMAD1 promoter activation in the indicated cells was assessed by dual-luciferase reporter assays. pGL3-BMP4 or pGL3-SMAD1 promoter luciferase reporter constructs containing or lacking the FOXF2-binding element were transfected into the indicated cells. e BMP reporter activation was assessed based on the fluorescence intensity of GFP. ${ }^{\star} P<0.05$ by Student's $t$ test. Error bars are defined as S.d. $\mathbf{f} B M P 4$ and SMAD1 mRNA levels in primary breast cancer tissues that developed bone-only metastasis $(B O M ; n=61)$ or metastases in other distant organs with or without bone metastasis (NBM; $n=36$ ) were analyzed based on the GSE2034_GSE2603 data set. $\mathbf{g}$ BMP4 and SMAD1 mRNA levels in metastatic tissues in bone (BM; $n=18)$ and in other distant organs (NBM; $n=47)$ were analyzed based on the GSE14020 data set. $\mathbf{h}$, $\mathbf{i}$ Kaplan-Meier analysis of the BOMFS rate of patients with different BMP4 or SMAD1 mRNA levels (h) or combined FOXF2/BMP4 and FOXF2/SMAD1 mRNA levels (i) in primary breast cancers was performed based on the GSE2034_GSE2603 data set. ${ }^{*} P<0.05$ and ${ }^{* \star *} P<0.001$, FOXF2 high $/ B M P 4_{\text {high }}$ Vs. FOXF2 low $/ B M P 4_{\text {low }}$ and FOXF2 $2_{\text {high }} / S M A D 1_{\text {high }}$ VS.

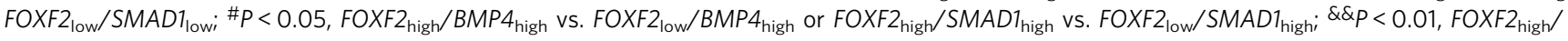
$S M A D 1_{\text {high }}$ vs. FOXF2 $2_{\text {high }} / S M A D 1_{\text {low }}$

To provide clinical evidence validating the role of CTSK and the FOXF2-CTSK axis in breast cancer bone metastasis, we analyzed CTSK expression levels in metastatic tissues in bone and other organs based on the GSE14020 data set. The results revealed that CTSK was highly expressed in bone metastatic tissues compared with metastatic tissues in other distant organs (Fig. 8h). We further analyzed the clinical relevance of CTSK or combined FOXF2/CTSK mRNA levels with bone-specific metastasis based on the GSE2034_GSE2603 data set. The results revealed that the rate of BOMFS in $C T S K_{\text {high }}$ patients is lower than that in $C T S K_{\text {low }}$ 
a

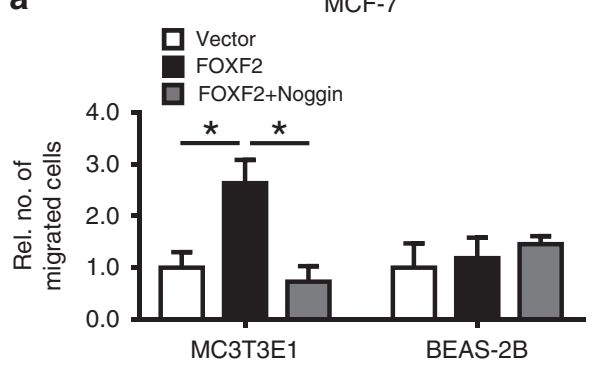

b

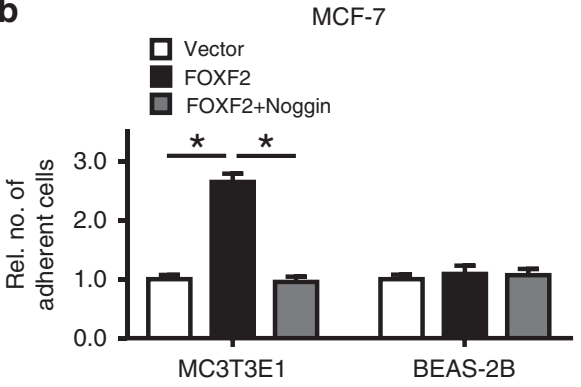

c

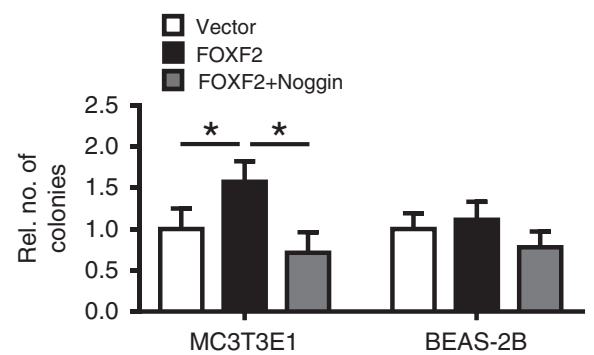

d
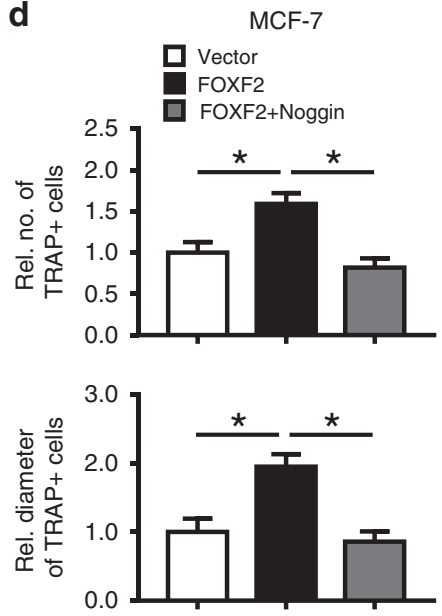

MDA-MB-231

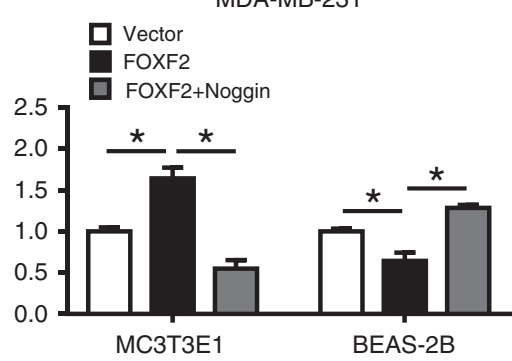

MDA-MB-231

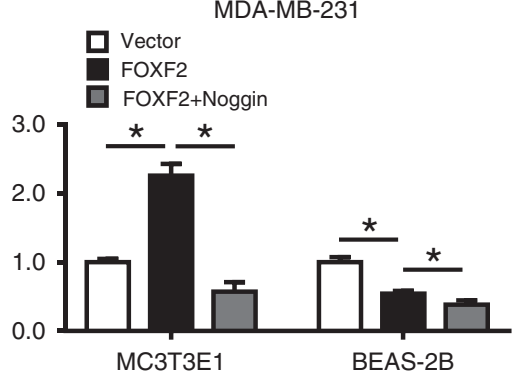

MDA-MB-231

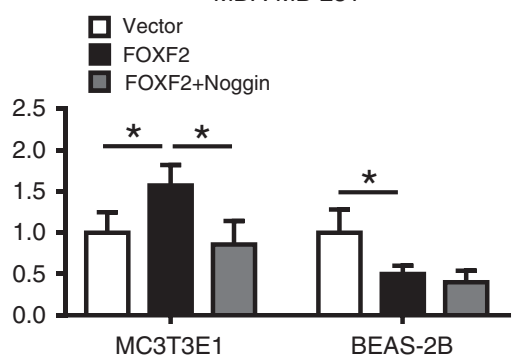

MDA-MB-231
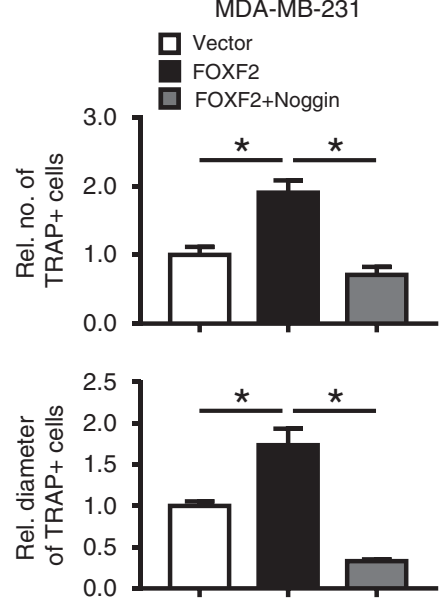

MDA-MB-231

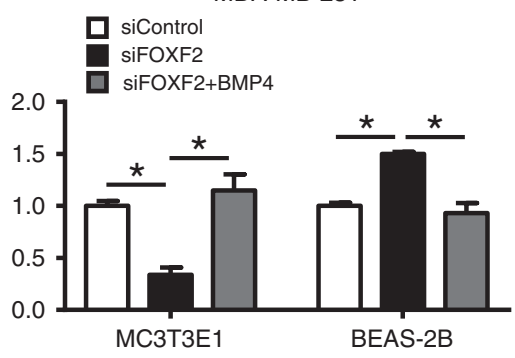

MDA-MB-231

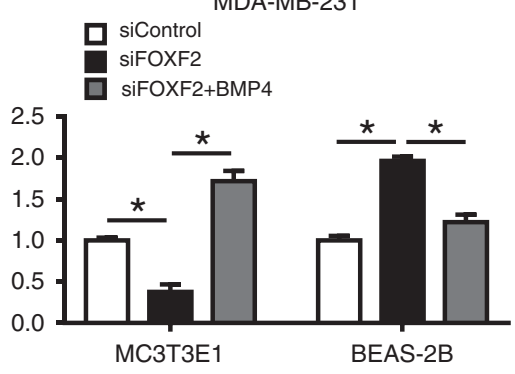

MDA-MB-231

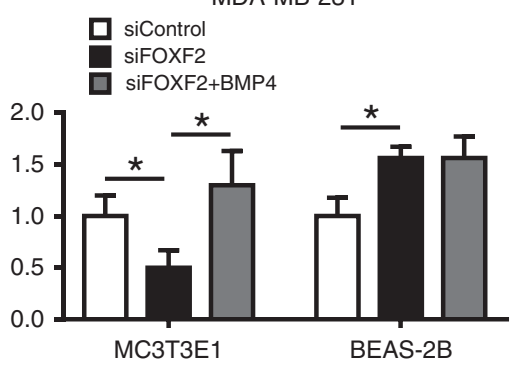

MDA-MB-231
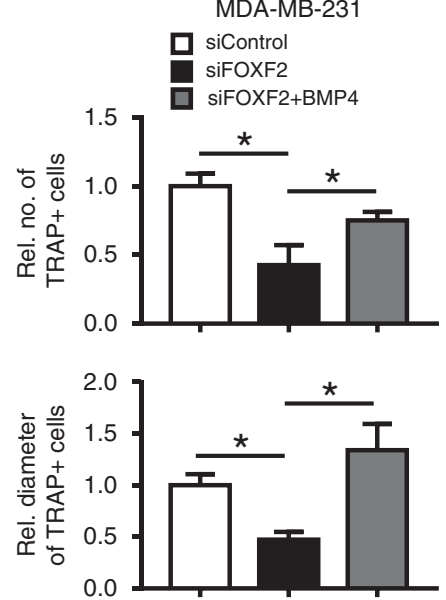

Fig. 6 FOXF2 enhances the bone-specific metastasis of breast cancer cells through activating the BMP/SMAD signaling pathway. MCF-7 and MDA-MB231 cells were treated as indicated. a Chemotactic migration of cancer cells toward MC3T3E1 or BEAS-2B cells was assessed by transwell assays. b Adhesion of cancer cells to MC3T3E1 or BEAS-2B cells was assessed by adding cancer cells to MC3T3E1 or BEAS-2B cells at $100 \%$ saturation, followed by incubation for $30 \mathrm{~min}$. c Anchorage-independent growth of cancer cells in the CM from MC3T3E1 or BEAS-2B cells was assessed by soft agar colonyformation assays. $\mathbf{d}$ The ability of cancer cells for induction of osteoclast differentiation was assessed by incubation of pre-induced primary preosteoclasts with cancer cell CM containing $50 \mathrm{ng} \mathrm{ml}^{-1}$ RANKL. The number and size of mature osteoclasts with TRAP-positive multinucleated ( $\geq 3$ nuclei) were analyzed. ${ }^{\star} P<0.05$ by Student's $t$ test. Error bars are defined as s.d.

patients, and $F O X F 2_{\text {high }} / C T S K_{\text {high }}$ patients had a worse BOMFS than patients in the FOXF2 $2_{\text {low }} / C T S K_{\text {high }}, F O X F 2_{\text {high }} / C T S K_{\text {low }}$, and $F O X F 2_{\text {low }} / C T S K_{\text {low }}$ groups (Fig. 8i). These results imply that the FOXF2-CTSK axis plays a critical role in breast cancer bonespecific metastasis.
Since CTSK is a known target of BMP/SMAD/RUNX2 $2^{20}$, we also investigated the presence of a BMP/SMAD signaling pathway-dependent mechanism for FOXF2-regulated CTSK expression by detecting CTSK and phospho-SMAD1/5 protein levels in MDA-MB-231 cells with FOXF2 knockdown plus BMP4 
a

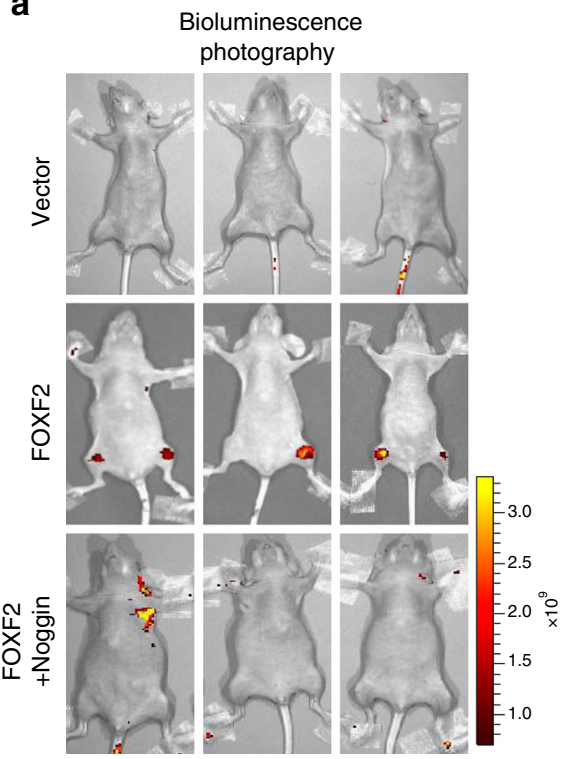

Day 49 after left ventricle injection

C

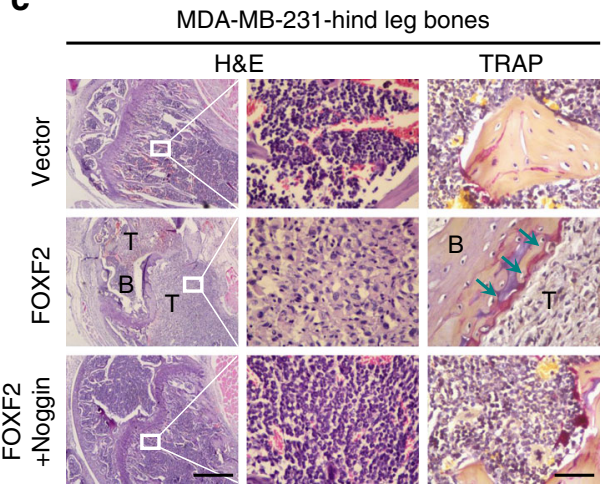

e

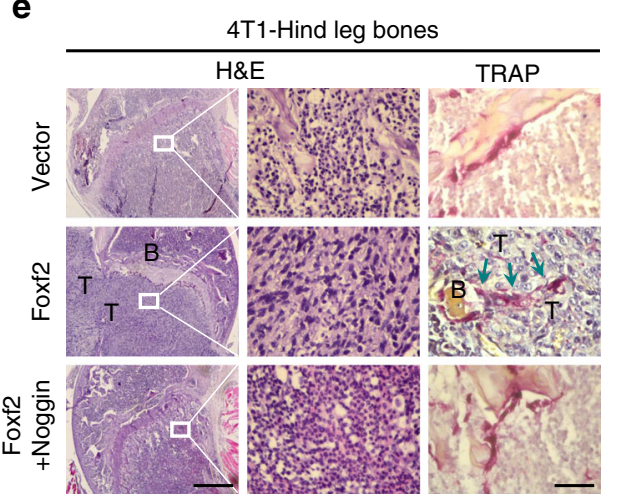

b

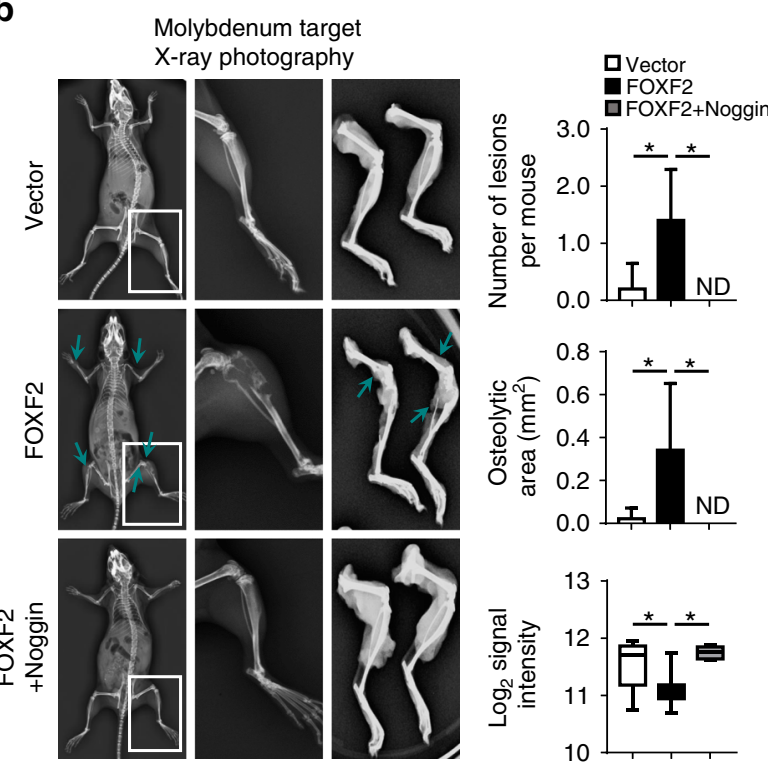

Day 49 after left ventricle injection
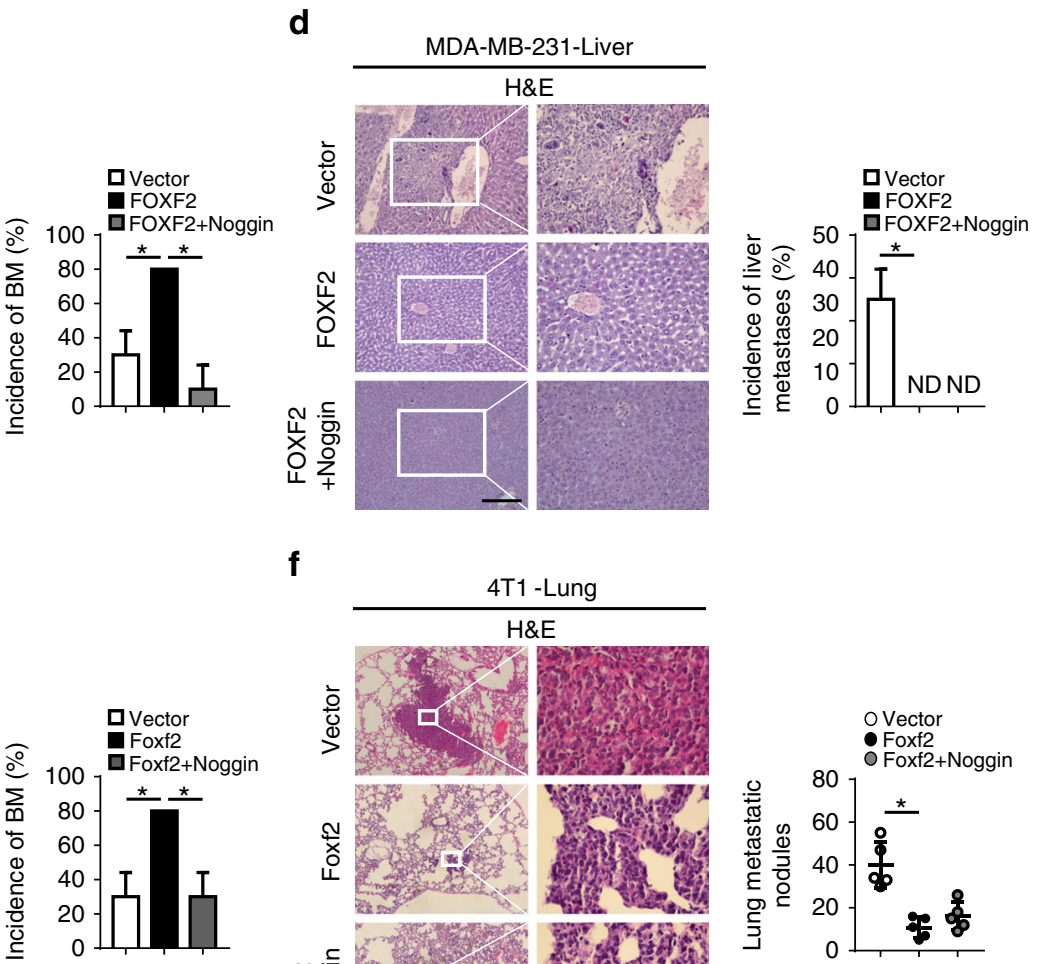

f
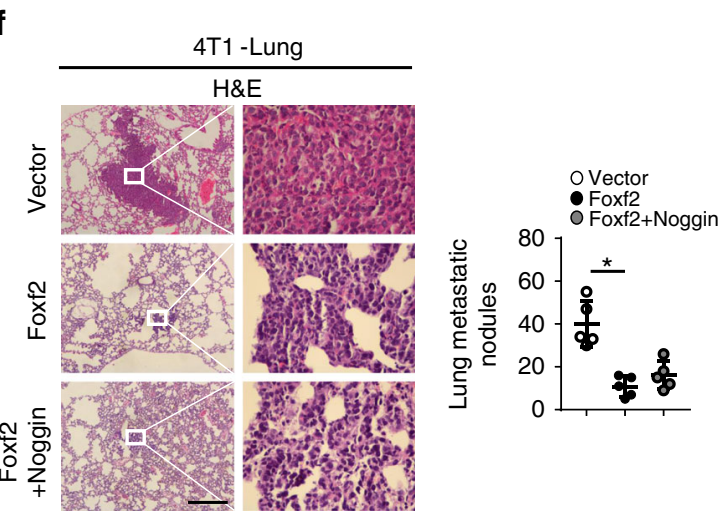

Fig. 7 FOXF2 enhances the bone-specific metastasis of breast cancer cells through activating the BMP/SMAD signaling pathway. A total of $1 \times 10^{5}$ MDAMB-231 cells or $2 \times 10^{5} 4 \mathrm{~T} 1$ cells infected with LV-FOXF2/Foxf2-EGFP (FOXF2/Foxf2) or LV-EGFP vector (Vector) were injected into the left ventricle or fat pad of female nude mice ( $n=5$ per group). The mice-bearing tumors overexpressing Foxf2 were treated with 500 ng of Noggin by intraperitoneal injection three times weekly for 3 (4T1) or 4 (MDA-MB-231) weeks. a, b Bioluminescence (a) and X-ray imaging (b) of mice injected with MDA-MB-231 cells treated as indicated were analyzed. Arrows point to osteolytic lesions. $\mathbf{c}$, $\mathbf{e}$ The metastases and osteoclasts in the hind leg bones of mice injected with MDA-MB-231 (c) or 4T1 (e) cells treated as indicated were visualized by H\&E and TRAP staining, respectively. Scale bars: $500 \mu \mathrm{m}$ for H\&E staining and $50 \mu \mathrm{m}$ for TRAP staining. Arrows point to osteolytic TRAP + osteoclasts. d, $\mathbf{f}$ Metastases in the liver (d) or lungs (f) of mice injected with MDA-MB-231 and $4 \mathrm{~T} 1$ cells treated as indicated were identified by H\&E staining. The metastatic nodules were counted, and the incidence of metastases was determined. Scale bars: $200 \mu \mathrm{m}$ for $(\mathbf{d})$ and $500 \mu \mathrm{m}$ for $(\mathbf{f}) .{ }^{\star} P<0.05$ by Student's $t$ test. Error bars are defined as s.d. 
a

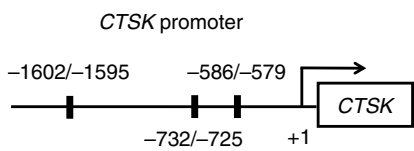

I Putative FOXF2 binding site b

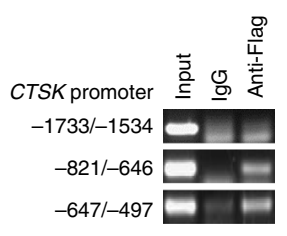

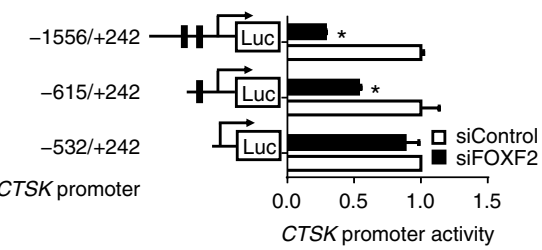

C

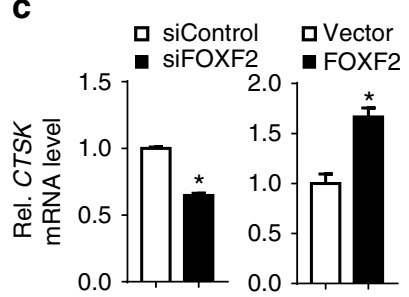

f

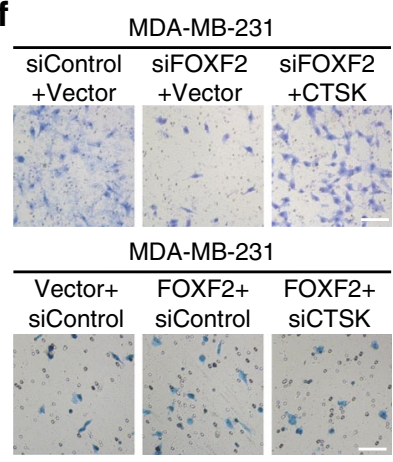

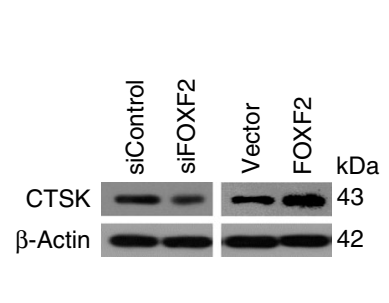

d

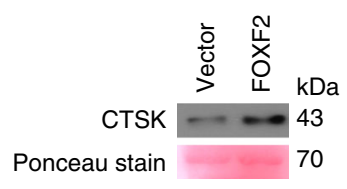

h

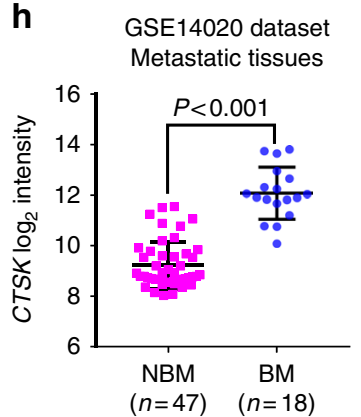

i

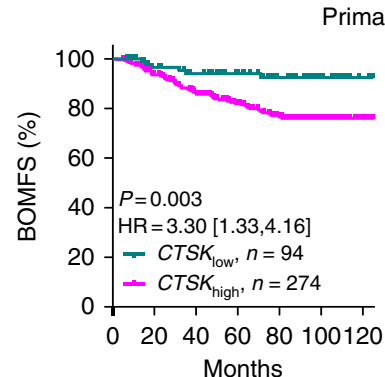

g

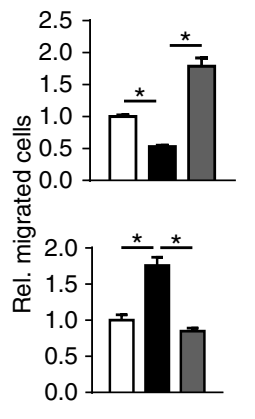

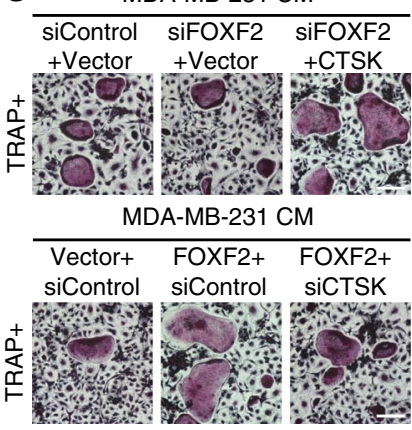

e Bone metastatic tissue

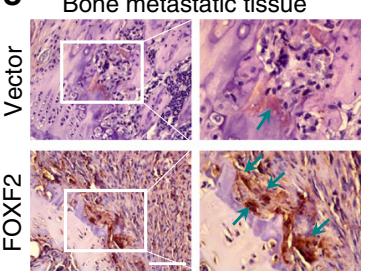

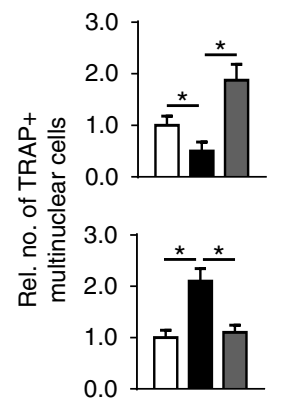

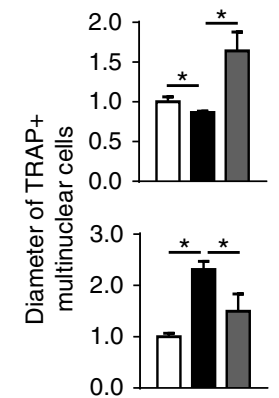

Fig. 8 FOXF2 enhances the osteolytic bone metastasis of breast cancer cells by transactivating CTSK expression. a Schematic of the candidate FOXF2binding sites in the CTSK promoter region (left) and the binding of FOXF2 to the CTSK promoter region containing the candidate-binding sites in MDA-MB231 cells transfected with pcDNA3.1-FOXF2-Flag was determined by ChIP assays using anti-Flag antibody or lgG control (right). b The transcriptional activity of the CTSK promoter in the indicated cells was assessed by dual-luciferase reporter assays. c CTSK mRNA (left) and intracellular protein (right) levels in MDA-MB-231 cells, treated as indicated, were detected by RT-qPCR and immunoblot. d CTSK secretion levels in CM from MDA-MB-231 cells, treated as indicated, were detected by immunoblot. e CTSK protein expression in bone metastatic tissues in mice-bearing MDA-MB-231 cells treated as indicated was detected by immunohistochemistry. Arrows point to CTSK + cells. $\mathbf{f}$ The bone-matrix degradation and invasive capacity of the indicated cells were assessed by transwell assays. The insert was coated with bone matrix generated by MC3T3E1 cells. $\mathbf{g}$ The ability of cancer cells to induce osteoclast differentiation was assessed by incubation of pre-induced primary preosteoclasts with cancer cell CM containing $50 \mathrm{ng} \mathrm{ml}^{-1} \mathrm{RANKL}$. The number and size of mature TRAP-positive multinucleated ( $\geq 3$ nuclei) osteoclasts were analyzed. Scale bars: $100 \mu \mathrm{m}$. ${ }^{\star} P<0.05$ by Student's $t$ test. Error bars are defined as s.d. $\mathbf{h}$ CTSK expression levels in metastatic tissues in bone $(n=18)$ and in other distant organs $(n=47)$ were compared based on the GSE14020 data set. $\mathbf{i}$ Kaplan-Meier analysis of BOMFS of patients with different CTSK (left) or combined FOXF2 and CTSK (right) mRNA levels in primary breast cancers was performed based on the GSE2034_GSE2603 data set. j CTSK and phospho-SMAD1/5 protein levels in MDA-MB-231 cells, treated as indicated, were detected by immunoblot

or with FOXF2 overexpression plus Noggin. The result revealed that FOXF2 positively regulated both CTSK and phosphoSMAD1/5 expression, and the BMP/SMAD signaling pathway mediated FOXF2-regulated CTSK expression (Fig. 8j). Thus, in addition to direct transcriptional regulation, FOXF2 also regulates CTSK expression through the BMP/SMAD signaling pathway.

\section{Discussion}

Cancer cells that have undergone EOT are transformed into bone metastasis seeds that preferentially home to the bone microenvironment $^{5}$, wherein they survive ${ }^{6}$ and colonize ${ }^{7,8}$. The fact that osteomimetic cancer cells commonly exist in breast cancer tissues $^{20}$ could explain the predilection of breast cancer bone 
metastasis, which has an incidence as high as $80 \%$ in advanced breast cancer pants ${ }^{21}$. The acquisition of osteomimetic features in cancer cells is considered similar to the differentiation of the osteoblastic lineage, a process involving the progression of mesenchymal stem cells to osteoprogenitors, osteoblasts, and ultimately cortical lining cells or osteocytes ${ }^{22}$. The signature of BRGs ectopically coexpressed in breast cancer tissues mainly includes genes encoding matrix proteins that are expressed at the early stages of bone differentiation, e.g., collagens, SPARC/OSN, and fibronectin (FN), but does not include the matrix proteins expressed at a nonreversible differentiation stage, e.g., alkaline phosphatase (ALP), or those expressed in mature osteoblasts and terminally differentiated osteocytes, e.g., bone sialoprotein (BSP), decorin, and osteocalcin (OC) ${ }^{20,22}$. This evidence indicates that the fate of osteomimetic cancer cells as bone metastasis seeds is determined by their status that is similar to osteoprogenitors or pre-osteoblast-harboring stem-like properties and reversible differentiation potential. Thus, osteomimetic cancer cells could gain invasive, disseminating, and osteotropic properties, which is dependent on reciprocal interaction between cancer cells and the microenvironment during metastasis.

FOXF2 is specifically expressed in the mesenchyme adjacent to the epithelium to maintain the characteristics of mesenchymal cells and inhibit the mesenchymal transformation of epithelial cells during embryonic development and tissue differentiation ${ }^{11}$. Our previous studies have demonstrated that FOXF2 is highly expressed in basal-like breast cells, but less in non-basal-like breast cells, while FOXF2 deficiency promotes early-onset visceral organ metastasis of BLBC cells through triggering epithelial-mesenchymal transition-inducing transcription factor (EMT-TF) networks ${ }^{12,13}$. In this study, we found that FOXF2 is ectopically coexpressed with a set of BRGs in primary breast cancer tissues. Although the set of BRGs includes both collagenous and noncollagenous proteins, the target genes potentially transregulated by FOXF2 encode noncollagenous proteins primarily expressed in osteoprogenitors or pre-osteoblasts, which differs from that potentially transregulated by RUNX2. RUNX2 directly regulates the transcription of both collagenous and noncollagenous matrix proteins encoded by BRGs highly expressed in osteotropic breast cancer ${ }^{20}$. Importantly, FOXF2 not only directly upregulates the transcription of BRGs but also directly regulates the transcription of genes encoding key signal and effecter molecules in the osteogenic differentiation signaling pathway, BMP4 and SMAD1. The BMP signaling pathway plays a key role in breast cancer bone metastasis ${ }^{23}$, and this process could be regulated by zinc-finger protein 217 (ZNF217) $)^{24}$. Our study not only extends the understanding of the mechanisms responsible for the osteomimetic and osteotropic phenotypes of cancer cells but also identified a determiner of cell differentiation fate toward an osteocyte lineage. Our data also suggest that FOXF2 mRNA level in combination with the mRNA levels of the FOXF2 target genes BMP4/SMAD1 or BRGs may be biomarkers for the prediction of breast cancer bone metastasis risk, and targeting the FOXF2-BMP/SMAD axis might be a promising therapeutic strategy to manage breast cancer bone metastasis.

CTSK is produced not only by osteoclasts for osteoclastmediated osteoclastic bone resorption ${ }^{25}$ but also by osteoblasts to maintain the collagenous matrix and recycle improperly processed collagen ${ }^{26}$. CTSK is ectopically expressed in breast cancer cells that metastasize to bone to promote tumor cell invasiveness and contribute to bone degradation ${ }^{27,28}$. CTSK inhibitor therapy reduced skeletal tumor burden but did not block the growth of primary tumor, indicating that CTSK generates a favorable bone microenvironment for tumor growth by promoting bone resorption ${ }^{28}$. However, the mechanism regulating CTSK expression remains to be investigated. Our group has reported that
CTSK is a direct transcriptional target of RUNX2, a master mediator of the BMP/SMAD signaling for bone development and remodeling, and that CTSK is coexpressed with a set of BRGs regulated by RUNX2 in primary breast cancer tissues ${ }^{20}$. In this study, we identified FOXF2 as another direct regulator of CTSK transcription and CTSK-mediated breast cancer bone metastasis. In addition to direct transcriptional regulation, FOXF2 also regulates CTSK expression through the BMP/SMAD/ RUNX2 signaling pathway. Our findings suggest that the FOXF2CTSK axis may be a potential target for the prevention and treatment of bone metastasis-caused osteolytic bone destruction.

In addition to the role of FOXF2 in promoting the bonespecific metastasis of breast cancer, we also observed a role of FOXF2 in suppressing the visceral organ metastasis of BLBC, which is consistent with our previous findings that FOXF2deficient BLBC cells have a propensity to metastasize to visceral organs $^{12,13}$. Recently, Zhuang et al. reported that the Wnt signaling inhibitor dickkopf (DKK1) played opposite roles in breast cancer bone and lung metastasis: promotion of bone metastasis and suppression of lung metastasis ${ }^{29}$. Interestingly, FOXF2 acts as an inhibitor of the Wnt signaling pathway ${ }^{17,30}$, which implies that the opposite roles of FOXF2 in BLBC bone and visceral organ metastasis may also involve inhibition of the Wnt signaling pathway. The subtype-specific and metastatic organ-specific patterns of FOXF2 expression and role in BLBC reflect the pleiotropic regulatory function of FOXF2 in BLBC development and metastasis.

Our previous studies indicated that FOXF2 deficiency accelerates the non-bone distant metastasis of BLBC through pleiotropic transactivation of the EMT-TFs TWIST1 ${ }^{12}$, FOXC2 ${ }^{13}$ and FOXQ1 ${ }^{31}$. In this study, we observed that FOXF2 negatively regulates $\mathrm{p}-\mathrm{SMAD} 2$ and $\mathrm{p}-\mathrm{SMAD} 3$, indicating that FOXF2 activates the $\mathrm{BMP} / \mathrm{SMAD}$ signaling pathway but inhibits the transforming frowth factor beta (TGF- $\beta$ )/SMAD pathway, a key inducer of EMT/mesenchymal stem cell properties ${ }^{32}$. Thus, antagonism between BMP/SMAD and TGF- $\beta /$ SMAD plays a critical role in the FOXF2-regulated organotropism of breast cancer metastasis. Our in vitro data suggest that ectopic expression of FOXF2 in luminal breast cancer cells not only promotes bone metastasis but also may enhance non-bone metastatic potential under conditions of stromal cell induction. However, we failed to observe any metastasis in the MCF-7-FOXF2/nude mouse ventricle injection model, and clinical data revealed that FOXF2 negatively correlated with non-bone metastasis in breast cancer cases (Supplementary Fig. 1b). In fact, TNBC/BLBC is most likely to develop visceral metastases within 3 years after diagnosis and surgery, while luminal breast cancer is more likely to metastasize to bone ${ }^{33-36}$. The role and mechanism of FOXF2 in regulating the non-bone distant metastasis of luminal breast cancer cells remain to be further investigated.

In summary, we provide mechanistic insights into breast cancer bone metastasis. We showed that the mesenchymal TF FOXF2, which is ectopically expressed in breast cancer cells, drives breast cancer cells to develop into bone metastasis seeds by directly programming the EOT. This study not only extends the role of FOXF2 in cancer but also proposes that the deregulation of pleiotropic TFs controlling embryonic development and tissue differentiation can lead to complex biological processes in cancer, which may underlie refractory cancer metastasis. In addition, the transactivation function of FOXF2 on BRGs implies a biological function of FOXF2 in regulating bone development and remodeling.

\section{Methods}

Tissue specimens. A total of 118 primary breast cancer tissues diagnosed as invasive ductal carcinoma were collected from breast cancer patients as described 
previously ${ }^{20}$. Among these cases, 15 developed bone metastasis and 18 suffered non-bone metastasis within a 10 -year follow-up period after primary tumor surgery. Tumors with estrogen receptor-positive $(\mathrm{ER}+) /$ progesterone receptorpositive $(\mathrm{PR}+)$ /human epidermal growth factor receptor 2-negative (HER2-) were classified as the luminal subtype, and ER-/PR-/HER2 - tumors were classified as the triple-negative subtype. The use of these specimens in this study was approved by the Institutional Review Board and the Research Ethics Committee of TMUCIH, and written consent was obtained from all participants.

Gene expression profiling data sets. Our gene expression profiling data set of 49 primary breast cancer tissues ${ }^{9}$ and GOBO tool were used to identify BRGs coexpressed with FOXF2 ${ }^{10}$. The GeneCards (http://www.genecards.org/) and NCBIPubMed (http://www.ncbi.nlm.nih.gov/pubmed/) databases were used to select BRGs that encode osteoblast-specific TFs, osteoblast-specific adhesion molecules, bone-matrix proteins, bone-matrix-degrading enzymes, and growth factors that regulate bone remodeling and bone-related disease ${ }^{20}$. The gene expression profiling data set in Gene Expression Omnibus (GEO) under accession number GSE20685 $5^{37}$ was used to validate the correlation between FOXF2 and BRGs in primary breast cancer tissues $(n=327)$. The combined gene expression profiling data set GSE12777_GSE15026_GSE65194 ${ }^{38-40}$ of breast cancer cell lines $(n=95)$ and the gene expression profiling data sets of primary breast cancer tissues in ArrayExpress (AE; https://www.ebi.ac.uk/arrayexpress/) under accession number E-MTAB-365 $5^{41}$ $(n=287)$ and in GEO under accession number GSE3494 ${ }^{42}(n=118)$ were used to compare the difference in FOXF2 expression levels between luminal and BLBC/ TNBC subtypes. The combined gene expression profiling data set GSE2034_GSE260343,44 $(n=368)$, which contains follow-up data on organs affected by metastasis, was used to analyze the expression of FOXF2 and BRGs in primary breast cancer tissues and the survival of patients with different affected organs based on FOXF2 and BRG expression. Among the 119 patients suffered from distant metastasis, 83 developed bone metastasis, among which 61 developed bone-only metastasis; 39 developed lung metastasis, among which 20 developed lung-only metastasis; 15 developed liver metastasis, among which 5 developed liver-only metastasis; and 15 developed brain metastasis, among which 5 developed brain-only metastasis. The GSE14020 data set ${ }^{45}$ was used to compare gene expression levels in different metastatic tissues $(n=65)$, including bone metastasis $(n=18)$, lung metastasis $(n=20)$, liver metastasis $(n=5)$, and brain metastasis $(n=22)$. Optimal cutoff values of FOXF2, SMAD1, and CTSK mRNA levels were determined based on receiver-operating characteristic (ROC) curves and the maximum Youden's index to separate all participants and different patient subgroups into high- and low-expression groups with distinct metastasis statuses.

Cells and treatment. The human TNBC/BLBC cell lines MDA-MB-231 (ATCC, Manassas, VA, USA), MDA-MB-231-Luc-BM (231-Luc-BM, a subline of MDAMB-231 cells expressing luciferase and with greater propensity for bone metastasis), and the mouse TNBC/BLBC cell line 4T1 (ATCC) were cultured in RPMI 1640 (Thermo Fisher Scientific, Carlsbad, CA, USA). The human luminal breast cancer cell line MCF-7 (ATCC) and immortalized lung epithelial cell line BEAS-2B (ATCC) were cultured in the Dulbecco's modified Eagle's Medium (Thermo Fisher Scientific). The mouse preosteoblastic cell line MC3T3E1 (ATCC) was cultured in Minimum Essential Medium-Alpha (Thermo Fisher Scientific). The osteoblast differentiation of MC3T3E1 cells was induced with $10 \mathrm{mM} \beta$-glycerol phosphate (Sigma, St. Louis, MO, USA) and $50 \mathrm{\mu g} \mathrm{ml}^{-1} \mathrm{~L}$-ascorbic acid 2-phosphate (Sigma) for 14 days. Primary HPFs (ScienCell, CA, USA) and HHSCs (ScienCell) were cultured in the fibroblast medium (ScienCell) and stellate cell medium (ScienCell), respectively. All cell lines were authenticated by short tandem repeat profiling and tested for mycoplasma contamination.

Cancer cells were cocultured with MC3T3E1 cells, BEAS-2B cells, HPFs, and HHSCs or their CM diluted with three volumes of fresh normal medium ${ }^{20}$. Noggin (500 ng ml-1; PeproTech Inc, Rocky Hill, NJ, USA) or recombinant human BMP4 ( $20 \mathrm{ng} \mathrm{ml}^{-1}$; Sigma) was added to the culture medium to treat cancer cells for $72 \mathrm{~h}$. Bovine serum albumin (for BMP4) or phosphate-buffered saline (for Noggin) was added at an equal volume in a parallel test as a control. All cell culture media were supplemented with $10 \%$ fetal bovine serum (FBS; Thermo Fisher Scientific), $100 \mathrm{U}$ $\mathrm{ml}^{-1}$ penicillin and $100 \mu \mathrm{g} \mathrm{ml}-1$ streptomycin, and cells were cultured at $37^{\circ} \mathrm{C}$ in a humidified incubator with $5 \% \mathrm{CO}_{2}$.

RT-qPCR. The total RNA in tissues and cells was extracted with TRIZOL reagent (Thermo Fisher Scientific) following the manufacturer's instructions. Reverse transcription (RT) of the total RNA was performed using SuperScript ${ }^{\text {tm }}$ First-Strand Synthesis System (Thermo Fisher Scientific). Quantitative PCR (qPCR) was carried out using the Platinum ${ }^{\circledR}$ Quantitative PCR System (Thermo Fisher Scientific) or SYBR $^{\circ}$ Premix Ex Taq ${ }^{\mathrm{TM}}$ (TaKaRa, Dalian, China) using primers and probes listed in Supplementary Table 1. The relative expression level of the target gene was calculated by normalizing the averaged cycle threshold $(\mathrm{Ct})$ values of target gene to the averaged $\mathrm{Ct}$ values of housekeeping gene glyceraldehyde-3-phosphate dehydrogenase (GAPDH; $\triangle \mathrm{Ct}$ ), and determined as $2^{-\Delta \mathrm{Ct}}$.

Immunoblot. Cells were lysed with protein lysis buffer containing $20 \mathrm{mM}$ Tris- $\mathrm{HCl}$ $\mathrm{pH}$ 7.4, $5 \mathrm{mM}$ EDTA, $1 \%$ Triton-X 100, $150 \mathrm{mM} \mathrm{NaCl}, 1 \%$ DTT, and $1 \%$ protease inhibitor cocktail (Thermo Fisher Scientific). The proteins in the lysate were separated by SDS-PAGE and transferred to polyvinyldifluoride membranes (BioRad Laboratories, Hercules, CA, USA). The membranes were blocked in 5\% skimmed milk in TBST (10 mM Tris, $150 \mathrm{mM} \mathrm{NaCl}, 0.05 \%$ Tween 20 , pH 8.3) for $1 \mathrm{~h}$ at room temperature, and then incubated with a primary antibody in $5 \%$ skimmed milk in TBST at $4{ }^{\circ} \mathrm{C}$ overnight. The following day, the membranes were washed in TBST and then incubated with a HRP-conjugated secondary antibody for $45 \mathrm{~min}$ at room temperature. The membranes were then washed in TBST, and immunoreactive protein bands were visualized by enhanced chemiluminescence reagents (Millipore, Billerica, MA, USA). The detailed information of primary and secondary antibodies is described in Supplementary Table 2.

Immunohistochemistry. Immunohistochemistry staining of formalin-fixed and paraffin-embedded tissue specimens was carried out using a primary antibody and a peroxidase-conjugated secondary antibody with appropriate concentration. Visualization of proteins was performed with diaminobenzidine and hematoxylin was used as counterstaining. The primary antibodies were anti-phospho-SMAD1/5 (Cell Signaling Technology, cat. 9516; 1:200) and anti-CTSK (Santa Cruz, cat. 48353; 1:200).

Plasmids and small interfering RNA transfection. Three siFOXF2 and siCTSK were synthesized by RiboBio Co. (Guangzhou, China), and the two with the best knockdown efficiency, as determined by RT-qPCR and immunoblot, were selected for transient FOXF2 knockdown experiments. siControl was used as a control. FOXF2 and CTSK cDNAs were inserted into the pcDNA3.1-HA, pcDNA3.1-Flag, or pcDNA3.1 vector. Plasmid and siRNA transfections were performed using Lipofectamine 2000 (Thermo Fisher Scientific), according to the manufacturer's instructions.

Lentivirus infection. Lentiviruses containing fusions of EGFP cDNA with human or mouse full-length FOXF2/Foxf2 (LV-FOXF2/Foxf2-EGFP), short-hairpin RNA targeting FOXF2/Foxf2 (LV-shFOXF2/shFoxf2-EGFP), or their controls were constructed by GeneChem Co., Ltd, Shanghai, China or Cyagen Biosciences Inc, China. MDA-MB-231 and 4T1 cells were infected with LV-FOXF2/Foxf2-EGFP, LV-shFOXF2/shFoxf2-EGFP, or their controls. The cells with stable overexpression or knockdown of FOXF2/Foxf2 were selected by puromycin and identified by RTqPCR and immunoblot.

Chemotactic migration assay. Chemotactic migration of breast cancer cells toward a mimic organ microenvironment was assessed using transwell inserts (8$\mu \mathrm{m}$ pore size; BD Biosciences, CA, USA) in a 24-well plate. MC3T3E1 cells, BEAS2B cells, HPFs, or HHSCs were pre-seeded in the lower chamber with the culture medium containing 10\% FBS. Cancer cells transfected with FOXF2 plasmid, siFOXF2\#1/\#2, or their controls were seeded in the upper chamber, and were allowed to migrate for an appropriate time. The non-migrating cells on the upper surface of the membrane were removed by wiping. The migrating cells were fixed with $4 \%$ paraformaldehyde, stained with a Rapid Wight-Giemsa Staining Kit (BBI Life Sciences, Shanghai, China), and counted under a microscope in at least three predetermined fields for each chamber at $\times 200$ magnification.

Heterogeneous cell-cell adhesion assay. Cancer cells transfected with GFPlabeled FOXF2 plasmid, siFOXF2\#1/\#2, or their controls were seeded on preinoculated MC3T3E1 cells, BEAS-2B cells, HPFs, or HHSCs that were grown to nearly $100 \%$ confluence in a 24 -well plate for the appropriate time. The nonadherent cancer cells were removed. The adherent cells were fixed with $4 \%$ paraformaldehyde and counted under a microscope in at least three predetermined fields for each well at $\times 200$ magnification.

Soft agar colony-formation assay. Cancer cells were suspended in $0.3 \%$ agarose in the CM from MC3T3E1 cells, BEAS-2B cells, HPFs, or HHSCs with $10 \%$ FBS and placed on top of the $0.6 \%$ agarose gel in the serum-free normal medium in a six-well plate. The cells were cultured for 14 days and fed twice a week with fresh CM. Cell colonies greater than $50 \mu \mathrm{m}$ in diameter were counted.

Osteoclastogenesis assay. Primary preosteoclasts were isolated from bone marrow cells of 6-week-old wild-type BALB/c mice and cultured overnight in the minimum Eagle's medium with $10 \%$ FBS. Non-adherent cells were plated in a 12 well plate supplemented with $30 \mathrm{ng} \mathrm{ml}^{-1}$ macrophage colony-stimulating factor (M-CSF; PeproTech) and cultured for 3 days, and then, $50 \mathrm{ng} \mathrm{ml}^{-1}$ receptor activator of nuclear factor- $\kappa$ B ligand (RANKL; PeproTech) was added and the cells were incubated for 2 days. Finally, the CM from cancer cell was added, and the cells were incubated for an additional 7 days. Multinuclear cells were stained with TRAP. The number and size of mature osteoclasts with TRAP-positive multinucleated ( $\geq 3$ nuclei; TRAP + ) were scored.

Bone-matrix invasion assay. The osteoblast differentiation of MC3T3E1 cells was induced on transwell inserts. The cells were lysed by a solution containing $0.05 \%$ 
Triton-X-100 and $10 \mathrm{mM} \mathrm{NH}_{4} \mathrm{OH}$, and the bone matrix remained on the insert. Cancer cells were seeded on the bone-matrix-coated insert and allowed to invade toward $10 \%$ FBS for an appropriate time. The invading cells were counted under a microscope in at least three predetermined fields for each chamber at $\times 200$ magnification.

ChIP-PCR assay. ChIP assay was carried out by using a ChIP Assay Kit (Millipore) according to the manufacturer's instructions. Briefly, MCF-7 or MDA-MB231 cells transfected with FOXF2-Flag plasmids were cross-linked with $1 \%$ formaldehyde and resuspended in lysis buffer, then sonicated and incubated with an anti-Flag antibody. The protein-DNA complexes were enriched by packed beads with protein A agarose/Salmon sperm DNA. After elution and reversal, chromosomal DNA was purified by using a GeneJET PCR Purification Kit (Thermo Fisher Scientific), according to the manufacture's instruction. The proximal promoter regions of candidate FOXF2 target genes in the resulting DNA fragments were PCR amplified using primers listed in Supplementary Table 3.

Dual-luciferase reporter assay. The luciferase reporters of FOXF2 target gene proximal promoter region containing the FOXF2-binding element were constructs by amplification from the normal genomic DNA using primers listed in Supplementary Table 3 and subsequently inserted into the pGL3-basic vector (Promega, Madison, WI, USA). The promoter activation of FOXF2 target genes in MCF-7 or MDA-MB-231 cells was assessed by co-transfection with luciferase reporter constructs, internal control pRL-TK and siFOXF2, FOXF2, or their control. The relative promoter activation was represented as the ratio of firefly to Renilla luciferase activity measured at $48 \mathrm{~h}$ post transfection using a dual-luciferase reporter assay system (Promega). The BMP-GFP Reporter plasmid (YeaSen, Shanghai, China) was used to examine activation of the BMP signaling pathway.

Animal experiments. 4T1 cells infected with LV-Foxf2-EGFP, LV-shFoxf2-EGFP, or their controls were injected into the lower right abdominal mammary fat pad of female nude mice $\left(2 \times 10^{5}\right.$ cells $)$ or BALB/c mice $\left(5 \times 10^{6}\right.$ cells $)$ aged $4-6$ weeks. A total of $1 \times 10^{5}$ MDA-MB-231 cells infected with LV-FOXF2-EGFP, LV-shFOXF2EGFP, or control or $2 \times 10^{5} 231$-Luc-BM cells infected with LV-shFOXF2 or control were injected into the left ventricle of female nude mice (Nanjing Biomedical Research Institute of Nanjing University, China) aged 4-6 weeks. To inhibit or activate the BMP signaling pathway, the mice were treated with $500 \mathrm{ng}$ of Noggin or $40 \mathrm{ng}$ of BMP4 by intraperitoneal injection three times weekly for 4 weeks, beginning 1 (4T1) or 2 (MDA-MB-231 and 231-Luc-BM) weeks after cancer cell injection. The primary tumors in mice injected with $4 \mathrm{~T} 1$ cells were monitored every 5 days by size measurements. Tumor volume was calculated using the formula (length $\times$ width $^{2}$ )/2. GFP bioluminescence in mice injected with MDAMB-231 cells was imaged using a Xenogen IVIS system (Caliper Life Sciences, Hopkinton, MA, USA). The osteolytic status of the hind-leg bones and/or the whole-body skeleton was observed by molybdenum target X-ray photography. The number of osteolytic lesions, the osteolytic area, and the $\log _{2}$ signal intensity in hind-leg bones were calculated. The primary tumors, hind-leg bones, lungs, and livers were surgically harvested from the mice at killing. Bones were decalcified with $10 \%$ EDTA for 2 weeks. The metastatic nodules on the lung surface were counted, and the incidence of bone metastases and liver metastases was calculated. All tissues were fixed in $4 \%$ neutral-buffered formalin and paraffin-embedded for H\&E histological staining and immunohistochemical staining. The metastasis nodules were observed visually and counted by histological examination of formalin-fixed and H\&E-stained tissues. Mature osteoclasts in metastatic bone lesions were identified by TRAP staining and quantified as the number of TRAP + cells adjacent to tumor cells per millimeter interface. All animal experiments were complied with relevant ethical regulations, and protocols were approved by the Animal Ethics Committee of TMUCIH.

Statistical analysis. Pearson's correlation analysis was used to assess the correlation of the mRNA expression between genes based on the gene expression profiling data sets and the RT-qPCR data of breast cancer tissues. The data have been converted by logarithmic transformation and follows a normal distribution. The chi-square test or Student's $t$ test was applied to evaluate the differences in FOXF2 mRNA expression between the luminal and basal-like/triple-negative subtypes of human breast cancer, the differences in BRG mRNA expression between metastatic tissues in bone and in other organs. Kaplan-Meier survival analysis and the log-rank test were used to compare the metastasis-free survival statuses of patients with different FOXF2 and BRG expression levels and hazard ratios. All in vitro and in vivo assays were performed as at least two independent experiments in triplicate, and the data are presented as the mean \pm standard deviation (s.d.). Student's $t$ test was used to compare the differences between the experimental group and the control group. $P<0.05$ was considered significant.

Reporting summary. Further information on research design is available in the Nature Research Reporting Summary linked to this article.

\section{Data availability}

The gene expression profiling data sets of breast cancer tissues or cell lines that support the findings of this study are available from the corresponding references. The source data underlying all figures and supplementary figures are provided as a Source Data file.

Received: 6 February 2018 Accepted: 8 May 2019

Published online: 20 June 2019

\section{References}

1. Thibaudeau, L. et al. Mimicking breast cancer-induced bone metastasis in vivo: current transplantation models and advanced humanized strategies. Cancer Metastas-. Rev. 33, 721-735 (2014).

2. Zhang, X. H. et al. Selection of bone metastasis seeds by mesenchymal signals in the primary tumor stroma. Cell 154, 1060-1073 (2013).

3. Kaplan, R. N., Rafii, S. \& Lyden, D. Preparing the "Soil": The premetastatic niche. Cancer Res. 66, 11089-11093 (2006).

4. Rucci, N. \& Teti, A. Osteomimicry: how tumor cells try to deceive the bone. Front Biosci. (Sch. Ed.) 2, 907-915 (2010).

5. Kapoor, P., Suva, L. J., Welch, D. R. \& Donahue, H. J. Osteoprotegrin and the bone homing and colonization potential of breast cancer cells. J. Cell Biochem. 103, 30-41 (2008).

6. Gordon, J. A., Sodek, J., Hunter, G. K. \& Goldberg, H. A. Bone sialoprotein stimulates focal adhesion-related signaling pathways: role in migration and survival of breast and prostate cancer cells. J. Cell Biochem. 107, 1118-1128 (2009).

7. Tamura, D., Hiraga, T., Myoui, A., Yoshikawa, H. \& Yoneda, T. Cadherin-11mediated interactions with bone marrow stromal/osteoblastic cells support selective colonization of breast cancer cells in bone. Int. J. Oncol. 33, 17-24 (2008)

8. Li, X. Q., Lu, J. T., Tan, C. C., Wang, Q. S. \& Feng, Y. M. RUNX2 promotes breast cancer bone metastasis by increasing integrin alpha5-mediated colonization. Cancer Lett. 380, 78-86 (2016)

9. Feng, Y. et al. Evidence for a transcriptional signature of breast cancer. Breast Cancer Res. Treat. 122, 65-75 (2010).

10. Ringner, M., Fredlund, E., Hakkinen, J., Borg, A. \& Staaf, J. GOBO: gene expression-based outcome for breast cancer online. PloS One 6, el7911 (2011).

11. Aitola, M., Carlsson, P., Mahlapuu, M., Enerback, S. \& Pelto-Huikko, M. Forkhead transcription factor FoxF2 is expressed in mesodermal tissues involved in epithelio-mesenchymal interactions. Dev. Dynam. 218, 136-149 (2000).

12. Wang, Q. S., Kong, P. Z., Li, X. Q., Yang, F. \& Feng, Y. M. FOXF2 deficiency promotes epithelial-mesenchymal transition and metastasis of basal-like breast cancer. Breast Cancer Res.: BCR 17, 30 (2015).

13. Cai, J. et al. FOXF2 suppresses the FOXC2-mediated epithelial-mesenchymal transition and multidrug resistance of basal-like breast cancer. Cancer Lett. 367, 129-137 (2015).

14. Yu, Z. H. et al. Dual function of MAZ mediated by FOXF2 in basal-like breast cancer: Promotion of proliferation and suppression of progression. Cancer Lett. 402, 142-152 (2017).

15. Wang, Q. S. et al. FOXF2 deficiency permits basal-like breast cancer cells to form lymphangiogenic mimicry by enhancing the response of VEGF-C/ VEGFR3 signaling pathway. Cancer Lett. 420, 116-126 (2018).

16. van der Heul-Nieuwenhuijsen, L., Dits, N., Van Ijcken, W., de Lange, D. \& Jenster, G. The FOXF2 pathway in the human prostate stroma. Prostate 69, 1538-1547 (2009).

17. Nik, A. M., Reyahi, A., Ponten, F. \& Carlsson, P. Foxf2 in intestinal fibroblasts reduces numbers of $\operatorname{Lgr} 5(+)$ stem cells and adenoma formation by inhibiting Wnt signaling. Gastroenterology 144, 1001-1011 (2013).

18. Kundu, S. T. et al. The miR-200 family and the miR-183 96 182 cluster target Foxf2 to inhibit invasion and metastasis in lung cancers. Oncogene 35, 173-186 (2016).

19. van der Heul-Nieuwenhuijsen, L., Dits, N. F. \& Jenster, G. Gene expression of forkhead transcription factors in the normal and diseased human prostate. BJU Int. 103, 1574-1580 (2009).

20. Tan, C. C. et al. Breast cancer cells obtain an osteomimetic feature via epithelial-mesenchymal transition that have undergone BMP2/ RUNX2 signaling pathway induction. Oncotarget 7, 79688-79705 (2016).

21. Kozlow, W. \& Guise, T. A. Breast cancer metastasis to bone: mechanisms of osteolysis and implications for therapy. J. Mammary Gland Biol. Neoplasia 10, 169-180 (2005).

22. Koeneman, K. S., Yeung, F. \& Chung, L. W. Osteomimetic properties of prostate cancer cells: a hypothesis supporting the predilection of prostate cancer metastasis and growth in the bone environment. Prostate 39, 246-261 (1999). 
23. Katsuno, Y. et al. Bone morphogenetic protein signaling enhances invasion and bone metastasis of breast cancer cells through Smad pathway. Oncogene 27, 6322-6333 (2008).

24. Bellanger, A. et al. The critical role of the ZNF217 oncogene in promoting breast cancer metastasis to the bone. J. Pathol. 242, 73-89 (2017).

25. Morko, J. et al. Overexpression of cathepsin $\mathrm{K}$ accelerates the resorption cycle and osteoblast differentiation in vitro. Bone 44, 717-728 (2009).

26. Mandelin, J. et al. Human osteoblasts produce cathepsin K. Bone 38, 769-777 (2006).

27. Littlewood-Evans, A. J. et al. The osteoclast-associated protease cathepsin $\mathrm{K}$ is expressed in human breast carcinoma. Cancer Res. 57, 5386-5390 (1997)

28. Le Gall, C. et al. A cathepsin $\mathrm{K}$ inhibitor reduces breast cancer induced osteolysis and skeletal tumor burden. Cancer Res. 67, 9894-9902 (2007).

29. Zhuang, X. et al. Differential effects on lung and bone metastasis of breast cancer by Wnt signalling inhibitor DKK1. Nat. Cell Biol. 19, 1274-1285 (2017).

30. Ormestad, M. et al. Foxf1 and Foxf2 control murine gut development by limiting mesenchymal Wnt signaling and promoting extracellular matrix production. Development 133, 833-843 (2006).

31. Kang, L. J. et al. Reciprocal transrepression between FOXF2 and FOXQ1 controls basal-like breast cancer aggressiveness. FASEB J. 33, 6564-6573 (2019).

32. Mani, S. A. et al. The epithelial-mesenchymal transition generates cells with properties of stem cells. Cell 133, 704-715 (2008).

33. Smid, M. et al. Subtypes of breast cancer show preferential site of relapse. Cancer Res. 68, 3108-3114 (2008).

34. Soni, A. et al. Breast Cancer Subtypes Predispose the Site of Distant Metastases. Am. J. Clin. Pathol. 143, 471-478 (2015).

35. Kennecke, H. et al. Metastatic Behavior of Breast Cancer Subtypes. J. Clin. Oncol. 28, 3271-3277 (2010).

36. Bartmann, C. et al. Pattern of metastatic spread and subcategories of breast cancer. Arch. Gynecol. Obstet. 295, 211-223 (2017).

37. Kao, K. J., Chang, K. M., Hsu, H. C., Huang, A. T. Correlation of microarraybased breast cancer molecular subtypes and clinical outcomes: implications for treatment optimization. BMC cancer 11, 1-15 (2011).

38. Hoeflich, K. P. et al. In vivo antitumor activity of MEK and phosphatidylinositol 3-kinase inhibitors in basal-like breast cancer models. Clin. Cancer Res. 15, 4649-4664 (2009).

39. Mori, S. et al. Anchorage-independent cell growth signature identifies tumors with metastatic potential. Oncogene 28, 2796-2805 (2009).

40. Maubant, S. et al. Transcriptome analysis of Wnt3a-treated triple-negative breast cancer cells. Plos ONE 10, 1-26 (2015).

41. Reme, T., Hose, D., Theillet, C. \& Klein, B. Modeling risk stratification in human cancer. Bioinformatics 29, 1149-1157 (2013).

42. Miller, L. D. et al. An expression signature for p53 status in human breast cancer predicts mutation status, transcriptional effects, and patient survival P Natl Acad. Sci. USA 102, 13550-13555 (2005).

43. Wang, Y. et al. Gene-expression profiles to predict distant metastasis of lymph-node-negative primary breast cancer. Lancet 365, 671-679 (2005).
44. Minn, A. J. et al. Genes that mediate breast cancer metastasis to lung. Nature 436, 518-524 (2005).

45. Zhang, X. H. et al. Latent bone metastasis in breast cancer tied to Srcdependent survival signals. Cancer Cell 16, 67-78 (2009).

\section{Acknowledgements}

This work was supported by the National Natural Science Foundation of China (Nos. $81272357,81472680,81672894$, and 81872403).

\section{Author contributions}

S.W., G.X.L., C.C.T. and Y.M.F. designed the experiments. S.W., G.X.L., C.C.T. and L.J.K. performed the experiments. R.H. performed the gene expression profiling data set analysis. S.W., G.X.L., C.C.T., R.H., L.J.K., X.Q.L., Q.S.W., P.F.L., Q.L.Z. and Y.M.F. analyzed the data. Y.M.F. conceived, coordinated and supervised the studies, and wrote the paper.

\section{Additional information}

Supplementary Information accompanies this paper at https://doi.org/10.1038/s41467019-10379-7.

Competing interests: The authors declare no competing interests.

Reprints and permission information is available online at http://npg.nature.com/ reprintsandpermissions/

Peer review information: Nature Communications thanks Philip Owens and other anonymous reviewer(s) for their contribution to the peer review of this work.

Publisher's note: Springer Nature remains neutral with regard to jurisdictional claims in published maps and institutional affiliations.

(c) Open Access This article is licensed under a Creative Commons Attribution 4.0 International License, which permits use, sharing, adaptation, distribution and reproduction in any medium or format, as long as you give appropriate credit to the original author(s) and the source, provide a link to the Creative Commons license, and indicate if changes were made. The images or other third party material in this article are included in the article's Creative Commons license, unless indicated otherwise in a credit line to the material. If material is not included in the article's Creative Commons license and your intended use is not permitted by statutory regulation or exceeds the permitted use, you will need to obtain permission directly from the copyright holder. To view a copy of this license, visit http://creativecommons.org/ licenses/by/4.0/.

(C) The Author(s) 2019 